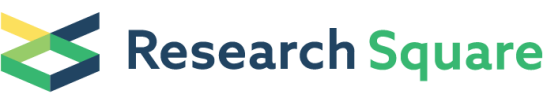 \\ Preprints are preliminary reports that have not undergone peer review. \\ They should not be considered conclusive, used to inform clinical practice, or referenced by the media as validated information.
}

\section{Uncovering the Potential Mechanism of Xue Fu Zhu Yu Decoction in the Treatment of Intracerebral Hemorrhage}

\section{Dao-jin Xue}

The Second Affiliated Hospital of Guangzhou University of Chinese Medicine

\section{Zheng Zhen}

Guangzhou University of Chinese Medicine

\section{Ke-xin Wang}

National Key Clinical Specialty/Engineering Technology Research Center of Education Ministry of China, Guangdong Provincial Key Laboratory on Brain Function Repair and Regeneration, Neurosurgery Insti Jia-lin Zhao

Guangdong Key Laboratory of Single Cell Technology and Application, Southern Medical University,

\section{Yao Gao}

First Hospital/First Clinical Medical College of Shanxi Medical University

\section{Yu-peng Chen}

Department of Biochemistry and Molecular Biology, School of Basic Medical Sciences, Southern

Medical University

\section{You-bi Shen}

The Second Affiliated Hospital of Guangzhou University of Chinese Medicine

\section{Zi-zhuang Peng}

The Second Affiliated Hospital of Guangzhou University of Chinese Medicine

\section{Dao-gang Guan}

Department of Biochemistry and Molecular Biology, School of Basic Medical Sciences, Southern Medical University

\section{Tao Huang ( $\nabla$ arteries@163.com )}

Guangzhou University of Chinese Medicine

\section{Research Article}

Keywords: Chinese herbal medicine (CHM), Intracerebral Hemorrhage (ICH), Important gene network model, Mechanism, Integrated pharmacology

Posted Date: January 7th, 2022

DOI: https://doi.org/10.21203/rs.3.rs-1157985/v1 
License: (c) (i) This work is licensed under a Creative Commons Attribution 4.0 International License. Read Full License

Version of Record: A version of this preprint was published at BMC Complementary Medicine and Therapies on April 12th, 2022. See the published version at https://doi.org/10.1186/s12906-022-03577-2. 


\section{Abstract \\ Background}

Chinese herbal medicine (CHM) is characterized by "multi- compounds, multi-targets and multi-pathway", which has advanced benefits for the preventing and treating complex diseases, but still exists unsolved issues, mainly include unclear material basis and underling mechanism of prescription. Integrated pharmacology is a hot cross research area based on system biology, mathematic and polypharmacology. It can systematically and comprehensively investigate the therapeutic reaction of compounds or drugs on pathogenic genes network, and is especially suitable for the study of complex $\mathrm{CHM}$ systems. Intracerebral Hemorrhage (ICH) is one of the main causes of death among Chinese residents, which is characterized by high mortality and high disability rate. In recent years, the treatment of ICH by CHM has been deeply researched. Xue Fu Zhu Yu Decoction (XFZYD), one of the commonly used prescriptions in treating $\mathrm{ICH}$ at clinic level, has not been clear about its mechanism in treating $\mathrm{ICH}$.

\section{Methods}

Here, we established a strategy, which based on compounds-targets, pathogenetic genes, network analysis and node importance calculation. Using this strategy, the core compounds group (CCG) of XFZYD was predicted and validated by in vitro experiments. The molecular mechanism of XFZYD in treating ICH was deduced based on CCG and their targets.

\section{Results}

The results show that the CCG with 43 compounds predicted by this model is highly consistent with the corresponding Compound-Target (C-T) network in terms of gene coverage, enriched pathway coverage and accumulated contribution of key nodes at $89.49 \%, 88.72 \%$ and $90.11 \%$, respectively, which confirmed the reliability and accuracy of the effective compound group optimization and mechanism speculation strategy proposed by us.

\section{Conclusions}

Our strategy of optimizing the effective compound groups and inferring the mechanism provides a strategic reference for explaining the optimization and inferring the molecular mechanism of prescriptions in treating complex diseases of $\mathrm{CHM}$.

\section{Introduction}

$\mathrm{ICH}$ refers to the hemorrhage caused by spontaneous and non-traumatic cerebral vascular rupture, which is often located in the deep brain tissues such as basal nucleus, putamen and thalamus, etc. It has the 
characteristics of acute onset, rapid change of disease condition, high mortality and disability rate, and is more common in middle-aged and elderly people. According to the statistical results, the prevalence rate of hemorrhagic stroke in China is 406.16/100,000, and the incidence rate is $26.34 / 100,000$ [ 1 ]. The proportion of cerebral hemorrhage in stroke patients in China $(24 \%)$ is higher than which in developed countries [ 2 ]. ICH is an important disease that seriously endangers the health and quality of life of middle-aged and elderly people. The main methods in treating ICH include drug therapy, surgical treatment, etc. Among them, controlling blood pressure is the most commonly used treatment based on drugs. In the early stage of the disease, the blood clots produced after bleeding will destroy the peripheral normal nerve cells, which will lead to the aggravation of neurological dysfunction such as hemiplegia and speech disorder. Therefore, how to promote hematoma absorption as soon as possible and reduce edema around the blood clots is a difficult point in conservative treatment.

In recent years, reports about treating $\mathrm{ICH}$ with $\mathrm{CHM}$ have gradually increased. For example, Buyang Huanwu Decoction can alleviate brain edema after ICH, reverse blood-brain barrier (BBB) damage and reduce nerve function damage [ 3 ]. Di Dang Tang can alleviate the nerve function injury and has better brain protection [ 4 ]. Xue Fu Zhu Yu Decoction (XFZYD) is the most widely used in ICH treatment. Clinical reports confirm that XFZYD combined with western medicine has a significant clinical effect in treating $\mathrm{ICH}$, which can improve the hypercoagulable state of blood in patients, thus promote the absorption of cerebral hematoma and reduce the neurological deficit [5]. Modern pharmacological experiments have also found that XFZYD can reduce the level of serum asym-metric dimethylarginine (ADMA) in atherosclerotic rats, thereby increasing the synthesis and secretion of NO, and further improving the pathological degree of atherosclerosis [ 6 ]. In addition, it can promote the secretion of vascular endothelial growth factor (VEGF) by human umbilical vein endothelial cells (HUVEC), suggesting that it may promote the proliferation of HUVEC by promoting the secretion of VEGF, thus playing a role in removing blood stasis and promoting new life [ 7 ].

The prescription contains 11 herbs: Rehmannia glutinosa (Gaertn.) (15g), Bupleurum chinense DC (5g), Glycyrrhiza uralensis Fisch (5g), Citrus aurantium L (10g). Platycodon grandiflorus (Jacq.) A.DC (7.5g), Achyranthes bidentata Blume(15g), Prunus alleghaniensis Porter $(20 \mathrm{~g})$, Carthamus tinctorius $(15 \mathrm{~g})$, Ligusticum chuanxiong S.H.Qiu (7.25g), Paeonia veitchii Lynch (10g), Angelica sinensis (Oliv.) (15g). Among these herbs, Prunus alleghaniensis Porterbreaks blood and moistens dryness, while Carthamus tinctorius $L$ promotes blood circulation and removes blood stasis to relieve pain. Paeonia veitchii $L y n c h$ and Ligusticum chuanxiong S.H.Qiu help the monarch to dispel blood stasis and promote blood circulation. Achyranthes bidentata Blume can enhance blood circulation, clear channels, remove blood stasis and relieve pain, and lead blood downward. Rehmannia glutinosa (Gaertn.) and Angelica sinensis (Oliv.) Diels are usually used to nourish blood and nourish yin, clear heat and promote blood circulation; Platycodon grandiflorus (Jacq.) A.DC. and Citrus aurantium $L$ can be used to disperse lung, relieve sore throat, eliminate phlegm and discharge pus; Bupleurum chinense $D C$ can be used to liver stagnation and qi stagnation, chest and rib pain, Glycyrrhiza uralensis Fisch has the efficacy of relieving pain and harmonizing various drugs. XFZYD composed of these herbs has the effects of eliminating edema and improving local circulation, so it is widely used in the treatment of $\mathrm{ICH}$. Although it has been reported, the 
mechanism is still unclear. How to find the key compounds to deduce the potential mechanism is the key step to understand the treatment of ICH by XFZYD.

At present, systematic pharmacology is a product of interdisciplinary research, including chemical and structural biology, bioinformatics, computer technology and mathematics, and also covers a large number of experimental disciplines including research technologies from cells, tissues to organs. In the recent researches, systematic pharmacology is widely used to explore the hidden mechanisms of $\mathrm{CHM}$ prescriptions on complex diseases. Such as, Wang Chun et al. built a contribution index model to analyze the molecular mechanism of Zhi-zhu Wan in treating Functional Dyspepsia [ 8 ]; Wang Kexin et al. quantified the molecular mechanism of Danggui Sini decoction (DSD), Guizhi Fuzi decoction (GFD), and Huangqi Guizhi Wuwu Decoction (HGWD), in treating rheumatoid arthritis based on the network communities detection model of network pharmacology [ 9 ]; Gao Yao et al. constructed optimization model based on network pharmacology to detect the molecular mechanisms of Lang Chuang Wan in treating systemic lupus erythematosus [ 10 ]. Increasing research evidence shows that systematic pharmacology has the characteristics of integrity and synergy in interpreting the underlying mechanisms of $\mathrm{CHM}$ prescription on complex diseases. Prescriptions in $\mathrm{CHM}$ has multi-compounds, multi-targets, multi-pathways mode of action in treating complex diseases, which reflects the system, integrity and coordination of $\mathrm{CHM}$, while the design idea of system pharmacology is consistent with these properties of prescriptions in the treatment process.

Systematic pharmacology of CHM provides novel ideas and perspectives for the study of complex CHM system. By using systematic pharmacology technology, the theories and methods of studying the active substances and combinations of $\mathrm{CHM}$, identifying the targets of pharmacodynamics compounds, and the relationship between pharmacodynamic substances and diseases were studied, so as to establish the pharmacodynamics of $\mathrm{CHM}$ and the basic theory of $\mathrm{CHM}$ based on systematic level. For example, Liu et al. used Network Pharmacology to prove the action of Jinshui Huanxian Formula on Idiopathic Pulmonary Fibrosis [ 11 ]. He et al. used systematic pharmacology to analyze the synergistic mechanism of LiuWei DiHuang Pill in Type 2 Diabetes Mellitus [ 12].

In this study, a new systems pharmacology model has been designed for capturing the key compounds and deducing the potential mechanism of XFZYD in treating $\mathrm{ICH}$. This model is based on novel node importance calculation method, and integrates compound monomer structure and target information, PPI network response mode and pathogenic genes reported in literature, and systematically explores the key effective compounds in treating ICH with XFZYD. The mechanism of CCG was deduced by using the target protein response score(TPRS) model. The reliability of the model is further confirmed by the verification of functional coverage and vitro experiments, which would provide methodological reference and advice for the systematic analysis of CHM in treating other complex diseases.

\section{Materials And Methods}




\subsection{Constructing the Weighted Pathogenic Gene-gene Interactions of ICH}

The protein-protein interaction (PPI) data was extracted from seven published database, include STRING[ 13 ], BioGRID, HPRD, Dip, Intact, Reactome and Mint. These data merged to construct comprehensive PPI network. ICH-related genes were extracted from Genecard, and genes with a relevance score higher than the average score were retained as highly pathogenic genes. These pathogenic genes were mapped into the PPI network to build the weighted pathogenic gene-gene interaction network of $\mathrm{ICH}$. Then the pathogenic gene-gene interactions were visualized by Cytoscape software (Version 3.7.2).

\subsection{Collectting Chemical Compounds of XFZYD}

All compounds of XFZYD were derived from three public herb medical data sources, TCMSP database [ 14 ], TCM integrated database [ 15] and TCM database@Taiwan [ 16 ]. The chemical properties of these compounds, include molecular weight (MW), oral bioavailability (OB), DL (drug-likeness) and Caco-2 permeability (Caco-2) were obtained from TCMSP. In addition, some compounds which were proved to with higher concentration in XFZYD in experiments were kept for next step analysis.

\subsection{Selectting Potential Active Compounds of XFZYD Based on ADME Models}

We use three ADME-related modules, $\mathrm{OB}, \mathrm{DL}$, and Caco-2 to screen biologically active compounds. $\mathrm{OB}$ stands for the radio of the oral constant-dose ingredient or drug that can be delivered to the circulation system [ 17]. Higher oral bioavailability usually is an important indicator to determine the drug-like property of biologically molecules as therapeutic drugs. We select suitable compounds with $\mathrm{OB} \geq 30 \%$ as active compounds for further analysis. The transport rates $(\mathrm{nm} / \mathrm{s})$ of compounds in Human intestinal cell line Caco-2 mono layers represent the intestinal epithelial permeability. The compounds with Caco-2>-0.4 were selected as the active compounds, because the compounds with a Caco- 2 value less than -0.4 were impermeable. Drug similarity helps optimize pharmacokinetics and drug properties, such as chemical stability and solubility. The "drug-like" level of 0.18 is usually used as a selection criterion for the "druglike" compounds in the CHM and was used in this study.

\subsection{Targets Prediction of Active compounds}

Three public databases, Similarity Ensemble Approach (SEA) [ 18 ], HitPick [ 19 ], and Swiss Target Prediction [ 20] were used in the targets prediction of active compounds in XFZYD. All chemical structures were converted into canonical SMILES by the software Open Babel toolkit (version 2.4.1).

\subsection{Constructing Node Importance Calculation Method}

For calculating the importance of each node in the network, we constructed a Node importance calculation method (BCR), in which, $n$ represent the number of nodes in the network; $s, v$ and $t$ represent nodes in the network; $V$ represents the collection of nodes within the network; $|\mathrm{V}|$ represents the number of 
nodes; $\sigma_{\mathrm{vt}}$ represents the number of the shortest path between nodes $\mathrm{v}$ and $\mathrm{t} ; \sigma_{\mathrm{vt}(\mathrm{s})}$ is the number of the shortest path passing through node $\mathrm{s} ; \mathrm{C}_{(\mathrm{s})}$ represents the genes which contains nodes; $\Delta_{\mathrm{C}(\mathrm{s})}$ is the maximum distance between gene $\mathrm{C}$ and other genes passing through node $s$; dist $(s, w)$ represents the length of a shortest path between nodes $s$ and $w$; The dist $(s, w)$ is equal to infinite if $C_{(s)} \neq C_{(w)}$, and it makes methods of this category cannot be applied to networks with disconnected genes. $\mathrm{BR}_{\mathrm{S}}$ represents the relationship between node $s$ and other nodes.

$$
\mathrm{BR}_{\mathrm{S}}=\sqrt{\left(\sum_{\mathrm{s} \neq \mathrm{v} \neq \mathrm{t} \in \mathrm{V}} \frac{\sigma_{\mathrm{vt}(\mathrm{s})}}{\sigma_{\mathrm{vt}}}\right) \times\left[\frac{\mid \mathrm{V}(\mathrm{C}(\mathrm{s})}{|\mathrm{V}|} \times \frac{\sum_{\mathrm{w} \in \mathrm{C}(\mathrm{s})}\left(\Delta_{\mathrm{C}(\mathrm{s})}+1-\operatorname{dist}_{(\mathrm{s}, \mathrm{w})}\right)}{\max \left\{\operatorname{dist}_{(\mathrm{s}, \mathrm{w})}: \mathrm{w} \in \mathrm{C}(\mathrm{s})\right\}}\right]}
$$

There are $\mathrm{n}$ nodes in the network. After being quantized, BR was sorted from small to large, and was represented by a new variable $\mathrm{G}$.

$$
\mathrm{G}=\left[\mathrm{G}_{1}, \mathrm{G}_{2}, \mathrm{G}_{3}, \ldots, \mathrm{G}_{\mathrm{n}}\right]=\left[\mathrm{BR}_{\mathrm{i}}, \mathrm{BR}_{\mathrm{i}+1}, \mathrm{BR}_{\mathrm{i}+2}, \ldots, \mathrm{BR}_{\mathrm{i}+\mathrm{m}}\right], \mathrm{x} \in[1, \mathrm{n}] \text { andi }+\mathrm{m}=\mathrm{n}
$$

The new variable P represented the nodes in the network. Each P responds to its unique G.

$$
P \in\left\{P_{1}, P_{2}, P_{3}, \ldots, P_{n}\right\} \rightleftharpoons\left[G_{1}, G_{2}, G_{3}, \ldots, G_{n}\right]
$$

BCR represented the important nodes selected form all nodes in the network. $\mathrm{N}$ represented natural number.

$$
B C R \in\left\{P_{r}, \ldots, P_{(n-2)}, P_{(n-1)}, P_{n}\right\} \rightleftharpoons\left[\frac{\left(G_{1}+G_{n}\right)}{2}, \ldots, G_{2}, G_{3}, \ldots, G_{n}\right], n \in N
$$

\subsection{Functional Annotations}

For analyzing the targets of XFZYD at the functional level, Gene Ontology (GO) analysis were performed by clusterProfiler package of R software with p-values of 0.05 [ 21 ], Kyoto Encyclopedia of Genes and Genomes (KEGG) enrichment analyses were constructed base on KEGG database with p-values of 0.05 [ 22 ]. Graphs were created by ggplot2 package in $\mathrm{R}$ language (version 3.4.2).

\subsection{Experimental Validation 2.8.1 Materials}

Acacetin, queretin, luteolin ( $\geq 98 \%$ purity by HPLC) was obtained from Jiangsu Yongjian Biotech Co., Ltd (Chengdu, China). Fetal bovine serum (FBS) and Dulbecco's modifified Eagle's medium (DMEM) were purchased from Gibco (Grand Island, USA). The mouse hippocampal HT22 cells were obtained from CHI 
SCIENTIFIC (Shanghai, China). Hypoxic bags were purchased from Mitsubishi Gas Chemical Company, Inc (Japanese). Cell Counting Kit-8 (CCK-8) was purchased from Dojindo Laboratories (Japanese).

\subsubsection{Cell Culture and Oxygen-glucose Deprivation (OGD) Treatment}

HT22 cells were cultured in DMEM with $10 \% \mathrm{FBS}$, and incubated at $37^{\circ} \mathrm{C}$ under $5 \% \mathrm{CO}_{2}$. Hypoxic bags were used to perform OGD model according to the method which had been reported [ 23 ].

\subsubsection{Cell Viability Assay}

HT22 cells $\left(6 \times 10^{4}\right.$ cells/well) were treated with different concentrations of acacetin, queretin, luteolin (40, $80,120,160$ and $200 \mu \mathrm{M}$ ) in 96-well plates. The plate reader was utilized to detect the absorbance at 450 $\mathrm{nm}$ after that CCK8 was superinduced for $4 \mathrm{~h}$.

\subsubsection{Statistical Analysis}

All data were expressed as mean \pm SEM. The differences were analyzed by one-way ANOVA for multiple comparisons, and Student's $t$ test was utilized to compare the significance of differences between two groups. Results were considered as statistically significant if the $p$-value was $<0.05$.

\section{Results}

\subsection{The Overview of Our Proposed Integrated Pharmacology Model}

Here, we designed a novel integrated pharmacology module to predict the CCG and depict the underlying mechanisms of XFZYD on ICH (Figures 1). In the first step, all XFZYD compounds were extracted from database and literatures. Next, the active compounds were figured out from all compounds of XFZYD based on proposed ADME models. Then we use three online tools to predict the targets of these active compounds. The weighted pathogenic gene-gene interactions and active compounds-targets network were merged to build the high reliable effective space (HRES) for decoding the effective proteins. TPRS was developed to determine the CCG. Finally, the CCG and their targets were used to figure out the underlying mechanisms of XFZYD in treating ICH.

\subsection{Construction of Weighted Pathogenic Gene-Gene Interaction Network for Hypertensive Cerebral Hemorrhage}

Weighted pathogenic gene-gene interaction network plays an important role in disease occurrence, drug response and other life activities. Constructing and analyzing weighted pathogenic gene-gene interaction network is the foundation in understanding the pathogenesis of $\mathrm{ICH}$ and can provide intervention strategies. To construct a weighted pathogenic gene-gene interaction network of ICH, PPI data were extracted and integrated from public databases, such as BioGRID, STRING, Dip, HPRD, Mint and 
Reactome. 863 genes were extracted from GeneCards related to $\mathrm{ICH}$, and each gene has a correlation score. If the correlation score is higher than the average score of these genes, it will be retained and used to build a weighted pathogenic gene-gene interaction network. There are many topological properties that can be used to describe the network, among which the degree is one of the indicators to describe the importance of nodes in the network. Through network topology analysis, it is found that APP, SRC, MAPK1 and other genes have higher degrees. Zhu et al. found that amyloid precursor protein (APP) mutation can cause typical pathological changes of $A D$ and perivascular amyloid deposition [ 24 ]. Fu et al. showed that NOTCH3 gene mutation may cause cerebral hemorrhage by changing the structure and function of cerebral small vessels [ 25]. Wang Lisong et al. found that the expression of c-myc is related to oxidative stress and neuronal apoptosis after intracerebral hemorrhage [ 26 ]. Qian Hong et al. found that EGFR expression increased in brain tissue around hematoma after intracerebral hemorrhage, and EGFR gene silencing was helpful to inhibit the activation of astrocytes in rats, and its mechanism might be related to blocking STAT3 phosphorylation [ 27 ]. These results indicate that these genes may play an important role in the pathogenesis of $\mathrm{ICH}$.

\subsection{Chemical Composition Collection and Composition Analysis}

Chemical identification is the critical step to clarify the material basis and action mechanism of compound prescription. In this study, the information of specific chemical compounds of XFZYD Chinese herbal medicines was obtained from literatures(Table 1). The results showed that the chemical compounds of herbs and the content of identified compounds provided experimental auxiliary chemical space for searching active compounds. The analysis of chemical constituents provides a reference for the screening of active constituents in XFZYD. 
Table 1

The chemical information of the herbs in XFZYD from the literature.

\section{Herb Method Component Concentration Ref.}

\begin{tabular}{|c|c|c|c|c|}
\hline \multirow{3}{*}{$\begin{array}{l}\text { Glycyrrhiza uralensis Fisch. ex DC. } \\
\text { (Gancao) }\end{array}$} & \multirow[t]{3}{*}{ HPLC } & Glycyrrhizin & $97.49 \mathrm{mg} / \mathrm{g}$ & \multirow{3}{*}{$\begin{array}{l}\text { Chen et } \\
\text { al., } 2009\end{array}$} \\
\hline & & Liquiritin & $\begin{array}{l}102.83 \\
\mathrm{mg} / \mathrm{g}\end{array}$ & \\
\hline & & Lsoliquritigenin & $98.30 \mathrm{mg} / \mathrm{g}$ & \\
\hline \multirow{7}{*}{$\begin{array}{l}\text { Angelica sinensis (Oliv.) Diels } \\
\text { (Danggui) }\end{array}$} & HPLC & Ferulic acid & $0.36 \mathrm{mg} / \mathrm{g}$ & \multirow{7}{*}{$\begin{array}{l}\text { Xie et al. } \\
2007\end{array}$} \\
\hline & & Coniferylferulate & $6.11 \mathrm{mg} / \mathrm{g}$ & \\
\hline & & Z-lig & $4.34 \mathrm{mg} / \mathrm{g}$ & \\
\hline & & ustilide & & \\
\hline & & E-ligustilide & $0.23 \mathrm{mg} / \mathrm{g}$ & \\
\hline & & Z-3-butylidenephthalide & $0.20 \mathrm{mg} / \mathrm{g}$ & \\
\hline & & E-3-butylidenephthalide & $0.08 \mathrm{mg} / \mathrm{g}$ & \\
\hline \multirow[t]{9}{*}{ Paeonia lactiflora Pall. (Chishao) } & \multirow{9}{*}{$\begin{array}{l}\text { RP- } \\
\text { HPLC }\end{array}$} & Gallic acid & $2.33 \mathrm{mg} / \mathrm{g}$ & \multirow{9}{*}{$\begin{array}{l}\text { Li et al., } \\
2011\end{array}$} \\
\hline & & Hydroxyl-paeoniflorin & $1.89 \mathrm{mg} / \mathrm{g}$ & \\
\hline & & Catechin & $0.03 \mathrm{mg} / \mathrm{g}$ & \\
\hline & & Albiflorin & $4.44 \mathrm{mg} / \mathrm{g}$ & \\
\hline & & Paeoniflorin & $4.81 \mathrm{mg} / \mathrm{g}$ & \\
\hline & & Benzoic acid & $0.03 \mathrm{mg} / \mathrm{g}$ & \\
\hline & & $\begin{array}{l}1,2,3,4,6- \\
\text { pentagalloylglucose }\end{array}$ & $4.80 \mathrm{mg} / \mathrm{g}$ & \\
\hline & & Benzoyl-paeoniflorin & $0.11 \mathrm{mg} / \mathrm{g}$ & \\
\hline & & Paeonol & $0.07 \mathrm{mg} / \mathrm{g}$ & \\
\hline \multirow[t]{6}{*}{ Citrus $\times$ aurantium L.(Zhike) } & \multirow[t]{6}{*}{ HPLC } & Naringenin & $1.91 \mathrm{mg} / \mathrm{g}$ & \multirow{6}{*}{$\begin{array}{l}\text { Zhan et } \\
\text { al., } \\
2015\end{array}$} \\
\hline & & Hesperetin & $1.37 \mathrm{mg} / \mathrm{g}$ & \\
\hline & & Marmin & $1.52 \mathrm{mg} / \mathrm{g}$ & \\
\hline & & $\begin{array}{l}\text { 6',7'-Dihydroxy } \\
\text { bergamot }\end{array}$ & $2.96 \mathrm{mg} / \mathrm{g}$ & \\
\hline & & Citronella & $2.90 \mathrm{mg} / \mathrm{g}$ & \\
\hline & & Orange peel & $2.18 \mathrm{mg} / \mathrm{g}$ & \\
\hline
\end{tabular}




\begin{tabular}{|c|c|c|c|c|}
\hline \multicolumn{5}{|c|}{ Herb Method Component Concentration Ref. } \\
\hline \multirow{4}{*}{$\begin{array}{l}\text { Prunus alleghaniensis } \\
\text { Porter(Taoren) }\end{array}$} & \multirow[t]{4}{*}{ HPLC } & cytidine & $0.43 \mathrm{mg} / \mathrm{g}$ & \multirow{4}{*}{$\begin{array}{l}\text { Li et al., } \\
2013\end{array}$} \\
\hline & & uridine & $0.29 \mathrm{mg} / \mathrm{g}$ & \\
\hline & & guanosine & $0.14 \mathrm{mg} / \mathrm{g}$ & \\
\hline & & adenosine & $0.79 \mathrm{mg} / \mathrm{g}$ & \\
\hline \multirow[t]{5}{*}{ Carthamus tinctorius L.(Honoghua) } & \multirow[t]{5}{*}{ HPLC } & Gallic acid & $3.37 \mathrm{mg} / \mathrm{g}$ & \multirow{5}{*}{$\begin{array}{l}\text { Wu et al. } \\
2019\end{array}$} \\
\hline & & hydroxysafflor yellow A & $97.92 \mathrm{mg} / \mathrm{g}$ & \\
\hline & & protocatechuic acid & $34.19 \mathrm{mg} / \mathrm{g}$ & \\
\hline & & rutin & $83.66 \mathrm{mg} / \mathrm{g}$ & \\
\hline & & quercetin & $19.51 \mathrm{mg} / \mathrm{g}$ & \\
\hline \multirow{3}{*}{$\begin{array}{l}\text { Ligusticum } \\
\text { chuanxiong S.H.Qiu(Chuangxiong) }\end{array}$} & \multirow[t]{3}{*}{ HPLC } & ferulic acid & $2.51 \mathrm{mg} / \mathrm{g}$ & \multirow{3}{*}{$\begin{array}{l}\text { Liu et al., } \\
2014\end{array}$} \\
\hline & & $\begin{array}{l}\text { Ligusticum wallichii } \\
\text { lactone }\end{array}$ & $0.7 \mathrm{mg} / \mathrm{g}$ & \\
\hline & & Ligustilide & $2.15 \mathrm{mg} / \mathrm{g}$ & \\
\hline \multirow{2}{*}{$\begin{array}{l}\text { Rehmannia glutinosa (Gaertn.) DC. } \\
\text { (Dihuang) }\end{array}$} & \multirow[t]{2}{*}{ HPLC } & Catalpol & $2.3 \mathrm{mg} / \mathrm{g}$ & \multirow{2}{*}{$\begin{array}{l}\text { Wu } \\
., 2016\end{array}$} \\
\hline & & Ophiopogon glycoside & $0.51 \mathrm{mg} / \mathrm{g}$ & \\
\hline \multirow{3}{*}{$\begin{array}{l}\text { Platycodon grandiflorus (Jacq.) } \\
\text { A.DC.(Jiegeng) }\end{array}$} & \multirow{3}{*}{$\begin{array}{l}\text { RP- } \\
\text { HPLC }\end{array}$} & deapioplatycoside $\mathrm{E}$ & $4.16 \mu \mathrm{g} / \mathrm{mL}$ & \multirow{3}{*}{$\begin{array}{l}\text { Wang et } \\
\text { al., } \\
2011\end{array}$} \\
\hline & & platycoside E & $10.57 \mu \mathrm{g} / \mathrm{mL}$ & \\
\hline & & platycodin D3 & $81.6 \mu \mathrm{g} / \mathrm{mL}$ & \\
\hline \multirow{5}{*}{$\begin{array}{l}\text { Achyranthes bidentata } \\
\text { Blume(Niuxi) }\end{array}$} & \multirow[t]{5}{*}{ HPLC } & 25 R-inokosterone & $0.626 \mathrm{mg} / \mathrm{g}$ & \multirow{5}{*}{$\begin{array}{l}\text { Lang et } \\
\text { al., } \\
2019\end{array}$} \\
\hline & & 25S-inokosterone & $0.127 \mathrm{mg} / \mathrm{g}$ & \\
\hline & & ecdysterone & $0.274 \mathrm{mg} / \mathrm{g}$ & \\
\hline & & Ginsenoside R0 & $0.701 \mathrm{mg} / \mathrm{g}$ & \\
\hline & & 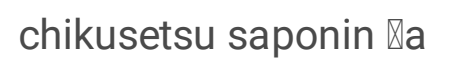 & 1. $798 \mathrm{mg} / \mathrm{g}$ & \\
\hline \multirow[t]{2}{*}{ Bupleurum chinense DC.(Chaihu) } & \multirow[t]{2}{*}{ HPLC } & \multirow[t]{2}{*}{ Saikosaponin D } & \multirow[t]{2}{*}{$0.016 \mathrm{mg} / \mathrm{g}$} & Hu et al., \\
\hline & & & & 2011 \\
\hline
\end{tabular}

\subsection{Selection of Active Compounds}

A total of 716 compounds were found in XFZYD through systematic search of the compounds in the published database of 11 herbs. Each CHM prescription contains hundreds of compounds, only a few compounds possess suitable pharmacokinetic and pharmacology dynamics properties. In the present 
work, three published ADME models, OB, Caco-2 and DL, were used to pick out the active compounds. After ADME screening, due to its high concentration and high biological activity, some compounds which did not meet the three screening criteria were also kept as the active compounds. Finally, 179 active compounds were selected from 716 compounds(Table 2). Butyl-phthalide is a potential active compound of XFZYD. Tu et al. found that butyl-phthalide can significantly reduce the neurological deficit in rats with intracerebral hemorrhage, which may be ascribed to butyl-phthalide by up-regulating the expression of VEGF and Ang-2 protein and increasing the density of neovascularization around hematoma without increasing the risk of hematoma enlargement. Quercetin is the main compound of $\mathrm{HL}$ and has a wide range of biological activities. Xu et al showed that early treatment with the best dose of quercetin could improve the brain injury after intracerebral hemorrhage model by inhibiting inflammatory reaction and reducing apoptosis [ 39 ], thus promoting the recovery of neural function. These results suggest that these compounds can treat ICH through multi-target synergistic mechanism. 
Table 2

Components in XFZYD after ADME screening.

\begin{tabular}{|c|c|c|c|c|c|}
\hline Herb & MOL_ID & molecule_name & ob & caco2 & $\begin{array}{l}\text { drug- } \\
\text { likeness }\end{array}$ \\
\hline NX & MOL012461 & 28-norolean-17-en-3-ol & 35.93 & 1.37 & 0.78 \\
\hline NX & MOL002714 & baicalein & 33.52 & 0.63 & 0.21 \\
\hline NX & MOL001454 & berberine & 36.86 & 1.24 & 0.78 \\
\hline NX & MOL000085 & beta-daucosterol_qt & 36.91 & 1.30 & 0.75 \\
\hline NX & MOL000358 & beta-sitosterol & 36.91 & 1.32 & 0.75 \\
\hline NX & MOL012505 & bidentatoside,ii_qt & 31.76 & -0.01 & 0.59 \\
\hline NX & MOL001458 & coptisine & 30.67 & 1.21 & 0.86 \\
\hline NX & MOL002643 & delta 7-stigmastenol & 37.42 & 1.30 & 0.75 \\
\hline NX & MOL002897 & epiberberine & 43.09 & 1.17 & 0.78 \\
\hline NX & MOL003847 & Inophyllum E & 38.81 & 0.68 & 0.85 \\
\hline NX & MOL000422 & kaempferol & 41.88 & 0.26 & 0.24 \\
\hline NX & MOL000785 & palmatine & 64.60 & 1.33 & 0.65 \\
\hline NX & MOL001006 & poriferasta-7,22E-dien-3beta-ol & 42.98 & 1.45 & 0.76 \\
\hline NX & MOL000098 & quercetin & 46.43 & 0.05 & 0.28 \\
\hline NX & MOL004355 & Spinasterol & 42.98 & 1.44 & 0.76 \\
\hline NX & MOL000449 & Stigmasterol & 43.83 & 1.44 & 0.76 \\
\hline NX & MOL000173 & wogonin & 30.68 & 0.79 & 0.23 \\
\hline DG & MOL008259 & 2,6-di(phenyl)thiopyran-4-thione & 69.13 & 1.74 & 0.15 \\
\hline DG & MOL000358 & beta-sitosterol & 36.91 & 1.32 & 0.75 \\
\hline DG & MOL000449 & Stigmasterol & 43.83 & 1.44 & 0.76 \\
\hline ZK & MOL000358 & beta-sitosterol & 36.91 & 1.32 & 0.75 \\
\hline ZK & MOL002341 & Hesperetin & 70.31 & 0.37 & 0.27 \\
\hline ZK & MOL013381 & Marmin & 38.23 & 0.14 & 0.31 \\
\hline ZK & MOL004328 & naringenin & 59.29 & 0.28 & 0.21 \\
\hline ZK & MOL005828 & nobiletin & 61.67 & 1.05 & 0.52 \\
\hline
\end{tabular}




\begin{tabular}{|c|c|c|c|c|c|}
\hline Herb & MOL_ID & molecule_name & $o b$ & caco2 & $\begin{array}{l}\text { drug- } \\
\text { likeness }\end{array}$ \\
\hline $\mathrm{HH}$ & MOL002694 & $\begin{array}{l}\text { 4-[(E)-4-(3,5-dimethoxy-4-oxo-1-cyclohexa-2,5- } \\
\text { dienylidene)but-2-enylidene]-2,6- } \\
\text { dimethoxycyclohexa-2,5-dien-1-one }\end{array}$ & 48.47 & 0.81 & 0.36 \\
\hline $\mathrm{HH}$ & MOL002712 & 6-Hydroxykaempferol & 62.13 & 0.16 & 0.27 \\
\hline $\mathrm{HH}$ & MOL002719 & 6-Hydroxynaringenin & 33.23 & 0.27 & 0.24 \\
\hline $\mathrm{HH}$ & MOL002757 & $\begin{array}{l}\text { 7,8-dimethyl-1H-pyrimido[5,6-g]quinoxaline- } \\
\text { 2,4-dione }\end{array}$ & 45.75 & 0.06 & 0.19 \\
\hline $\mathrm{HH}$ & MOL002714 & baicalein & 33.52 & 0.63 & 0.21 \\
\hline $\mathrm{HH}$ & MOL002773 & beta-carotene & 37.18 & 2.25 & 0.58 \\
\hline $\mathrm{HH}$ & MOL000358 & beta-sitosterol & 36.91 & 1.32 & 0.75 \\
\hline $\mathrm{HH}$ & MOL000953 & CLR & 37.87 & 1.43 & 0.68 \\
\hline $\mathrm{HH}$ & MOL000131 & EIC & 41.90 & 1.16 & 0.14 \\
\hline $\mathrm{HH}$ & MOL002680 & Flavoxanthin & 60.41 & 0.97 & 0.56 \\
\hline $\mathrm{HH}$ & MOL000422 & kaempferol & 41.88 & 0.26 & 0.24 \\
\hline $\mathrm{HH}$ & MOL002683 & Ligla & 45.01 & 1.20 & 0.15 \\
\hline $\mathrm{HH}$ & MOL002695 & lignan & 43.32 & 0.42 & 0.65 \\
\hline $\mathrm{HH}$ & MOL000432 & linolenic acid & 45.01 & 1.21 & 0.15 \\
\hline $\mathrm{HH}$ & MOL002698 & lupeol-palmitate & 33.98 & 1.52 & 0.32 \\
\hline $\mathrm{HH}$ & MOL000006 & luteolin & 36.16 & 0.19 & 0.25 \\
\hline $\mathrm{HH}$ & MOL001398 & Methyllinolenate & 46.15 & 1.48 & 0.17 \\
\hline $\mathrm{HH}$ & MOL000675 & oleic acid & 33.13 & 1.17 & 0.14 \\
\hline $\mathrm{HH}$ & MOL002706 & Phytoene & 39.56 & 2.22 & 0.50 \\
\hline $\mathrm{HH}$ & MOL002707 & phytofluene & 43.18 & 2.29 & 0.50 \\
\hline $\mathrm{HH}$ & MOL001771 & poriferast-5-en-3beta-ol & 36.91 & 1.45 & 0.75 \\
\hline $\mathrm{HH}$ & MOL002710 & Pyrethrin II & 48.36 & 0.53 & 0.35 \\
\hline $\mathrm{HH}$ & MOL002717 & qt_carthamone & 51.03 & -0.31 & 0.20 \\
\hline $\mathrm{HH}$ & MOL002721 & quercetagetin & 45.01 & -0.06 & 0.31 \\
\hline $\mathrm{HH}$ & MOL000098 & quercetin & 46.43 & 0.05 & 0.28 \\
\hline $\mathrm{HH}$ & MOL000449 & Stigmasterol & 43.83 & 1.44 & 0.76 \\
\hline
\end{tabular}




\begin{tabular}{|c|c|c|c|c|c|}
\hline Herb & MOL_ID & molecule_name & ob & caco2 & $\begin{array}{l}\text { drug- } \\
\text { likeness }\end{array}$ \\
\hline$C X$ & MOL000131 & EIC & 41.90 & 1.16 & 0.14 \\
\hline$c X$ & MOL002203 & Exceparl M-OL & 31.90 & 1.39 & 0.16 \\
\hline$c X$ & MOL001494 & Mandenol & 42.00 & 1.46 & 0.19 \\
\hline$C X$ & MOL001641 & METHYL LINOLEATE & 41.93 & 1.44 & 0.17 \\
\hline$c X$ & MOL002135 & Myricanone & 40.60 & 0.67 & 0.51 \\
\hline$c X$ & MOL000675 & oleic acid & 33.13 & 1.17 & 0.14 \\
\hline$c X$ & MOL002140 & Perlolyrine & 65.95 & 0.88 & 0.27 \\
\hline$c X$ & MOL002151 & senkyunone & 47.66 & 1.15 & 0.24 \\
\hline$c X$ & MOL000359 & sitosterol & 36.91 & 1.32 & 0.75 \\
\hline$c X$ & MOL002157 & wallichilide & 42.31 & 0.82 & 0.71 \\
\hline GC & MOL004941 & $\begin{array}{l}\text { (2R)-7-hydroxy-2-(4-hydroxyphenyl)chroman- } \\
\text { 4-one }\end{array}$ & 71.12 & 0.41 & 0.18 \\
\hline GC & MOL004805 & $\begin{array}{l}\text { (2S)-2-[4-hydroxy-3-(3-methylbut-2- } \\
\text { enyl)phenyl]-8,8-dimethyl-2,3- } \\
\text { dihydropyrano[2,3-f]chromen-4-one }\end{array}$ & 31.79 & 1.00 & 0.72 \\
\hline GC & MOL004824 & $\begin{array}{l}\text { (2S)-6-(2,4-dihydroxyphenyl)-2-(2- } \\
\text { hydroxypropan-2-yl)-4-methoxy-2,3- } \\
\text { dihydrofuro[3,2-g]chromen-7-one }\end{array}$ & 60.25 & 0.00 & 0.63 \\
\hline GC & MOL004945 & $\begin{array}{l}\text { (2S)-7-hydroxy-2-(4-hydroxyphenyl)-8-(3- } \\
\text { methylbut-2-enyl)chroman-4-one }\end{array}$ & 36.57 & 0.72 & 0.32 \\
\hline GC & MOL004815 & $\begin{array}{l}\text { (E)-1-(2,4-dihydroxyphenyl)-3-(2,2- } \\
\text { dimethylchromen-6-yl)prop-2-en-1-one }\end{array}$ & 39.62 & 0.66 & 0.35 \\
\hline GC & MOL004898 & $\begin{array}{l}\text { (E)-3-[3,4-dihydroxy-5-(3-methylbut-2- } \\
\text { enyl)phenyl]-1-(2,4-dihydroxyphenyl)prop-2-en- } \\
\text { 1-one }\end{array}$ & 46.27 & 0.41 & 0.31 \\
\hline GC & MOL004914 & $\begin{array}{l}\text { 1,3-dihydroxy-8,9-dimethoxy-6- } \\
\text { benzofurano[3,2-c]chromenone }\end{array}$ & 62.90 & 0.40 & 0.53 \\
\hline GC & MOL004913 & $\begin{array}{l}\text { 1,3-dihydroxy-9-methoxy-6-benzofurano[3,2- } \\
\text { c]chromenone }\end{array}$ & 48.14 & 0.48 & 0.43 \\
\hline GC & MOL005013 & 18a-hydroxyglycyrrhetic acid & 41.16 & -0.29 & 0.71 \\
\hline GC & MOL004959 & 1-Methoxyphaseollidin & 69.98 & 1.01 & 0.64 \\
\hline GC & MOL004866 & $\begin{array}{l}\text { 2-(3,4-dihydroxyphenyl)-5,7-dihydroxy-6-(3- } \\
\text { methylbut-2-enyl)chromone }\end{array}$ & 44.15 & 0.48 & 0.41 \\
\hline
\end{tabular}




\begin{tabular}{|c|c|c|c|c|c|}
\hline Herb & MOL_ID & molecule_name & ob & caco2 & $\begin{array}{l}\text { drug- } \\
\text { likeness }\end{array}$ \\
\hline GC & MOL004978 & $\begin{array}{l}\text { 2-[(3R)-8,8-dimethyl-3,4-dihydro-2H-pyrano[6,5- } \\
\text { f]chromen-3-yl]-5-methoxyphenol }\end{array}$ & 36.21 & 1.12 & 0.52 \\
\hline GC & MOL004849 & $\begin{array}{l}\text { 3-(2,4-dihydroxyphenyl)-8-(1,1-dimethylprop-2- } \\
\text { enyl)-7-hydroxy-5-methoxy-coumarin }\end{array}$ & 59.62 & 0.40 & 0.43 \\
\hline GC & MOL004863 & $\begin{array}{l}\text { 3-(3,4-dihydroxyphenyl)-5,7-dihydroxy-8-(3- } \\
\text { methylbut-2-enyl)chromone }\end{array}$ & 66.37 & 0.52 & 0.41 \\
\hline GC & MOL004905 & $\begin{array}{l}\text { 3,22-Dihydroxy-11-oxo-delta(12)-oleanene-27- } \\
\text { alpha-methoxycarbonyl-29-oic acid }\end{array}$ & 34.32 & -0.06 & 0.55 \\
\hline GC & MOL004966 & 3'-Hydroxy-4'-0-Methylglabridin & 43.71 & 1.00 & 0.57 \\
\hline GC & MOL004974 & 3'-Methoxyglabridin & 46.16 & 0.94 & 0.57 \\
\hline GC & MOL004864 & $\begin{array}{l}\text { 5,7-dihydroxy-3-(4-methoxyphenyl)-8-(3- } \\
\text { methylbut-2-enyl)chromone }\end{array}$ & 30.49 & 0.90 & 0.41 \\
\hline GC & MOL004989 & 6-prenylated eriodictyol & 39.22 & 0.40 & 0.41 \\
\hline GC & MOL004990 & 7,2',4'-trihydroxy-5-methoxy-3ロarylcoumarin & 83.71 & 0.24 & 0.27 \\
\hline GC & MOL004991 & 7-Acetoxy-2-methylisoflavone & 38.92 & 0.74 & 0.26 \\
\hline GC & MOL003896 & 7-Methoxy-2-methyl isoflavone & 42.56 & 1.16 & 0.20 \\
\hline GC & MOL004838 & $\begin{array}{l}\text { 8-(6-hydroxy-2-benzofuranyl)-2,2-dimethyl-5- } \\
\text { chromenol }\end{array}$ & 58.44 & 1.00 & 0.38 \\
\hline GC & MOL004993 & 8-prenylated eriodictyol & 53.79 & 0.43 & 0.40 \\
\hline GC & MOL000417 & Calycosin & 47.75 & 0.52 & 0.24 \\
\hline GC & MOL005020 & dehydroglyasperins C & 53.82 & 0.68 & 0.37 \\
\hline $\mathrm{GC}$ & MOL001792 & DFV & 32.76 & 0.51 & 0.18 \\
\hline $\mathrm{GC}$ & MOL004836 & echinatin & 66.58 & 0.38 & 0.17 \\
\hline $\mathrm{GC}$ & MOL004806 & euchrenone & 30.29 & 1.09 & 0.57 \\
\hline GC & MOL004915 & Eurycarpin A & 43.28 & 0.43 & 0.37 \\
\hline $\mathrm{GC}$ & MOL000392 & formononetin & 69.67 & 0.78 & 0.21 \\
\hline $\mathrm{GC}$ & MOL004996 & gadelaidic acid & 30.70 & 1.20 & 0.20 \\
\hline $\mathrm{GC}$ & MOL004856 & Gancaonin A & 51.08 & 0.80 & 0.40 \\
\hline GC & MOL004857 & Gancaonin B & 48.79 & 0.58 & 0.45 \\
\hline GC & MOL005000 & Gancaonin G & 60.44 & 0.78 & 0.39 \\
\hline
\end{tabular}




\begin{tabular}{|c|c|c|c|c|c|}
\hline Herb & MOL_ID & molecule_name & ob & caco2 & $\begin{array}{l}\text { drug- } \\
\text { likeness }\end{array}$ \\
\hline GC & MOL005001 & Gancaonin H & 50.10 & 0.60 & 0.78 \\
\hline GC & MOL004910 & Glabranin & 52.90 & 0.97 & 0.31 \\
\hline GC & MOL004911 & Glabrene & 46.27 & 0.99 & 0.44 \\
\hline GC & MOL004908 & Glabridin & 53.25 & 0.97 & 0.47 \\
\hline GC & MOL004912 & Glabrone & 52.51 & 0.59 & 0.50 \\
\hline GC & MOL004828 & Glepidotin A & 44.72 & 0.79 & 0.35 \\
\hline GC & MOL004829 & Glepidotin B & 64.46 & 0.46 & 0.34 \\
\hline GC & MOL004808 & glyasperin B & 65.22 & 0.47 & 0.44 \\
\hline GC & MOL004811 & Glyasperin C & 45.56 & 0.71 & 0.40 \\
\hline GC & MOL004810 & glyasperin F & 75.84 & 0.43 & 0.54 \\
\hline GC & MOL005007 & Glyasperins M & 72.67 & 0.49 & 0.59 \\
\hline GC & MOL004879 & Glycyrin & 52.61 & 0.59 & 0.47 \\
\hline GC & MOL002311 & Glycyrol & 90.78 & 0.71 & 0.67 \\
\hline GC & MOL005008 & Glycyrrhiza flavonol A & 41.28 & -0.09 & 0.60 \\
\hline GC & MOL004835 & Glypallichalcone & 61.60 & 0.76 & 0.19 \\
\hline GC & MOL004907 & Glyzaglabrin & 61.07 & 0.34 & 0.35 \\
\hline GC & MOL004957 & $\mathrm{HMO}$ & 38.37 & 0.79 & 0.21 \\
\hline GC & MOL004985 & icos-5-enoic acid & 30.70 & 1.22 & 0.20 \\
\hline GC & MOL001484 & Inermine & 75.18 & 0.89 & 0.54 \\
\hline GC & MOL004980 & Inflacoumarin A & 39.71 & 0.73 & 0.33 \\
\hline GC & MOL004948 & Isoglycyrol & 44.70 & 0.91 & 0.84 \\
\hline GC & MOL004949 & Isolicoflavonol & 45.17 & 0.54 & 0.42 \\
\hline GC & MOL001789 & isoliquiritigenin & 85.32 & 0.44 & 0.15 \\
\hline GC & MOL000354 & isorhamnetin & 49.60 & 0.31 & 0.31 \\
\hline GC & MOL004814 & Isotrifoliol & 31.94 & 0.53 & 0.42 \\
\hline GC & MOL000239 & Jaranol & 50.83 & 0.61 & 0.29 \\
\hline GC & MOL000422 & kaempferol & 41.88 & 0.26 & 0.24 \\
\hline
\end{tabular}




\begin{tabular}{|c|c|c|c|c|c|}
\hline Herb & MOL_ID & molecule_name & ob & caco2 & $\begin{array}{l}\text { drug- } \\
\text { likeness }\end{array}$ \\
\hline GC & MOL004988 & Kanzonol F & 32.47 & 1.18 & 0.89 \\
\hline GC & MOL004820 & kanzonols W & 50.48 & 0.63 & 0.52 \\
\hline GC & MOL005003 & Licoagrocarpin & 58.81 & 1.23 & 0.58 \\
\hline GC & MOL005012 & Licoagroisoflavone & 57.28 & 0.71 & 0.49 \\
\hline GC & MOL000497 & licochalcone a & 40.79 & 0.82 & 0.29 \\
\hline GC & MOL004841 & Licochalcone B & 76.76 & 0.47 & 0.19 \\
\hline GC & MOL004848 & licochalcone G & 49.25 & 0.64 & 0.32 \\
\hline GC & MOL004882 & Licocoumarone & 33.21 & 0.84 & 0.36 \\
\hline GC & MOL004885 & licoisoflavanone & 52.47 & 0.39 & 0.54 \\
\hline GC & MOL004883 & Licoisoflavone & 41.61 & 0.37 & 0.42 \\
\hline GC & MOL004884 & Licoisoflavone B & 38.93 & 0.46 & 0.55 \\
\hline GC & MOL004904 & licopyranocoumarin & 80.36 & 0.13 & 0.65 \\
\hline GC & MOL004855 & Licoricone & 63.58 & 0.53 & 0.47 \\
\hline GC & MOL003656 & Lupiwighteone & 51.64 & 0.68 & 0.37 \\
\hline GC & MOL000211 & Mairin & 55.38 & 0.73 & 0.78 \\
\hline GC & MOL002565 & Medicarpin & 49.22 & 1.00 & 0.34 \\
\hline GC & MOL004328 & naringenin & 59.29 & 0.28 & 0.21 \\
\hline GC & MOL005016 & Odoratin & 49.95 & 0.42 & 0.30 \\
\hline GC & MOL005017 & Phaseol & 78.77 & 0.76 & 0.58 \\
\hline GC & MOL004833 & Phaseolinisoflavan & 32.01 & 1.01 & 0.45 \\
\hline GC & MOL002844 & Pinocembrin & 64.72 & 0.61 & 0.18 \\
\hline GC & MOL000098 & quercetin & 46.43 & 0.05 & 0.28 \\
\hline GC & MOL004961 & Quercetin der. & 46.45 & 0.39 & 0.33 \\
\hline GC & MOL004827 & Semilicoisoflavone B & 48.78 & 0.45 & 0.55 \\
\hline GC & MOL004891 & shinpterocarpin & 80.30 & 1.10 & 0.73 \\
\hline GC & MOL004935 & Sigmoidin-B & 34.88 & 0.42 & 0.41 \\
\hline GC & MOL000359 & sitosterol & 36.91 & 1.32 & 0.75 \\
\hline
\end{tabular}




\begin{tabular}{|c|c|c|c|c|c|}
\hline Herb & MOL_ID & molecule_name & ob & caco2 & $\begin{array}{l}\text { drug- } \\
\text { likeness }\end{array}$ \\
\hline GC & MOL000500 & Vestitol & 74.66 & 0.86 & 0.21 \\
\hline GC & MOL005018 & Xambioona & 54.85 & 1.09 & 0.87 \\
\hline TR & MOL001328 & 2,3-didehydro GA70 & 63.29 & -0.27 & 0.50 \\
\hline TR & MOL000358 & beta-sitosterol & 36.91 & 1.32 & 0.75 \\
\hline TR & MOL000493 & campesterol & 37.58 & 1.31 & 0.71 \\
\hline TR & MOL000131 & EIC & 41.90 & 1.16 & 0.14 \\
\hline TR & MOL001339 & GA119 & 76.36 & -0.12 & 0.49 \\
\hline TR & MOL001340 & GA120 & 84.85 & 0.38 & 0.45 \\
\hline TR & MOL001342 & GA121-isolactone & 72.70 & -0.26 & 0.54 \\
\hline TR & MOL001343 & GA122 & 64.79 & -0.17 & 0.50 \\
\hline TR & MOL001344 & GA122-isolactone & 88.11 & -0.18 & 0.54 \\
\hline TR & MOL001358 & gibberellin 7 & 73.80 & -0.18 & 0.50 \\
\hline TR & MOL001351 & Gibberellin A44 & 101.61 & -0.13 & 0.54 \\
\hline TR & MOL000296 & hederagenin & 36.91 & 1.32 & 0.75 \\
\hline TR & MOL001371 & Populoside_qt & 108.89 & 0.49 & 0.20 \\
\hline TR & MOL001323 & Sitosterol alpha1 & 43.28 & 1.41 & 0.78 \\
\hline$J G$ & MOL001689 & acacetin & 34.97 & 0.67 & 0.24 \\
\hline$J G$ & MOL004580 & cis-Dihydroquercetin & 66.44 & -0.34 & 0.27 \\
\hline$J G$ & MOL000006 & luteolin & 36.16 & 0.19 & 0.25 \\
\hline$J G$ & MOL004355 & Spinasterol & 42.98 & 1.44 & 0.76 \\
\hline $\mathrm{CH}$ & MOL004653 & $(+)$-Anomalin & 46.06 & 0.46 & 0.66 \\
\hline $\mathrm{CH}$ & MOL004598 & $\begin{array}{l}\text { 3,5,6,7-tetramethoxy-2-(3,4,5- } \\
\text { trimethoxyphenyl)chromone }\end{array}$ & 31.97 & 0.75 & 0.59 \\
\hline $\mathrm{CH}$ & MOL004609 & Areapillin & 48.96 & 0.60 & 0.41 \\
\hline $\mathrm{CH}$ & MOL013187 & Cubebin & 57.13 & 0.47 & 0.64 \\
\hline $\mathrm{CH}$ & MOL000131 & EIC & 41.90 & 1.16 & 0.14 \\
\hline $\mathrm{CH}$ & MOL001789 & isoliquiritigenin & 85.32 & 0.44 & 0.15 \\
\hline $\mathrm{CH}$ & MOL000354 & isorhamnetin & 49.60 & 0.31 & 0.31 \\
\hline
\end{tabular}




\begin{tabular}{|c|c|c|c|c|c|}
\hline Herb & MOL_ID & molecule_name & ob & caco2 & $\begin{array}{l}\text { drug- } \\
\text { likeness }\end{array}$ \\
\hline $\mathrm{CH}$ & MOL000422 & kaempferol & 41.88 & 0.26 & 0.24 \\
\hline $\mathrm{CH}$ & MOL001645 & Linoleyl acetate & 42.10 & 1.36 & 0.20 \\
\hline $\mathrm{CH}$ & MOL004624 & Longikaurin A & 47.72 & 0.08 & 0.53 \\
\hline $\mathrm{CH}$ & MOL004683 & methyl (2E,4E)-octadeca-2,4-dienoate & 38.77 & 1.45 & 0.17 \\
\hline $\mathrm{CH}$ & MOL004628 & Octalupine & 47.82 & 0.48 & 0.28 \\
\hline $\mathrm{CH}$ & MOL000675 & oleic acid & 33.13 & 1.17 & 0.14 \\
\hline $\mathrm{CH}$ & MOL000490 & petunidin & 30.05 & 0.16 & 0.31 \\
\hline $\mathrm{CH}$ & MOL000098 & quercetin & 46.43 & 0.05 & 0.28 \\
\hline $\mathrm{CH}$ & MOL004702 & saikosaponin c_qt & 30.50 & 0.03 & 0.63 \\
\hline $\mathrm{CH}$ & MOL004644 & Sainfuran & 79.91 & 0.90 & 0.23 \\
\hline $\mathrm{CH}$ & MOL000449 & Stigmasterol & 43.83 & 1.44 & 0.76 \\
\hline $\mathrm{CH}$ & MOL004648 & Troxerutin & 31.60 & 0.35 & 0.28 \\
\hline $\mathrm{CH}$ & MOL004718 & a-spinasterol & 42.98 & 1.28 & 0.76 \\
\hline CS & MOL000492 & $(+)$-catechin & 54.83 & -0.03 & 0.24 \\
\hline CS & MOL006992 & $(2 \mathrm{R}, 3 \mathrm{R})-4$-methoxyl-distylin & 59.98 & 0.17 & 0.30 \\
\hline CS & MOL006994 & $\begin{array}{l}\text { 1-o-beta-d-glucopyranosyl-8-o- } \\
\text { benzoylpaeonisuffrone_qt }\end{array}$ & 36.01 & -0.03 & 0.30 \\
\hline CS & MOL006996 & 1-o-beta-d-glucopyranosylpaeonisuffrone_qt & 65.08 & -0.05 & 0.35 \\
\hline CS & MOL007008 & 4-ethyl-paeoniflorin_qt & 56.87 & -0.17 & 0.44 \\
\hline CS & MOL007012 & 4-o-methyl-paeoniflorin_qt & 56.70 & 0.40 & 0.43 \\
\hline CS & MOL007018 & 9-ethyl-neo-paeoniaflorin A_qt & 64.42 & -0.01 & 0.30 \\
\hline CS & MOL007005 & Albiflorin_qt & 48.70 & -0.38 & 0.33 \\
\hline CS & MOL002714 & baicalein & 33.52 & 0.63 & 0.21 \\
\hline CS & MOL000358 & beta-sitosterol & 36.91 & 1.32 & 0.75 \\
\hline CS & MOL000131 & EIC & 41.90 & 1.16 & 0.14 \\
\hline CS & MOL007022 & evofolinB & 64.74 & 0.00 & 0.22 \\
\hline CS & MOL001918 & paeoniflorgenone & 87.59 & -0.09 & 0.37 \\
\hline CS & MOL007016 & Paeoniflorigenone & 65.33 & -0.13 & 0.37 \\
\hline
\end{tabular}




\begin{tabular}{|llllll|}
\hline Herb & MOL_ID & molecule_name & ob & caco2 & $\begin{array}{l}\text { drug- } \\
\text { likeness }\end{array}$ \\
\hline CS & MOL001925 & paeoniflorin_qt & 68.18 & -0.34 & 0.40 \\
\hline CS & MOL000359 & sitosterol & 36.91 & 1.32 & 0.75 \\
\hline CS & MOL004355 & Spinasterol & 42.98 & 1.44 & 0.76 \\
\hline CS & MOL006999 & stigmast-7-en-3-ol & 37.42 & 1.32 & 0.75 \\
\hline CS & MOL000449 & Stigmasterol & 43.83 & 1.44 & 0.76 \\
\hline DH & MOL000131 & EIC & 41.90 & 1.16 & 0.14 \\
\hline DH & MOL003708 & jioglutin D & 39.02 & -0.22 & 0.14 \\
\hline DH & MOL000359 & sitosterol & 36.91 & 1.32 & 0.75 \\
\hline DH & MOL000449 & Stigmasterol & 43.83 & 1.44 & 0.76 \\
\hline
\end{tabular}

\subsection{Construction and Analysis of C-T Network}

In order to explore the mechanism of XFZYD in treating ICH, a C-T network was constructed with 179 active compounds and 838 targets. There are 6559 relationships among compounds and their targets because of that some active compounds can relate to multiple targets. The average number of targets of per compound is 54.55. The C-T network showed that XFZYD had the characteristics of multi-targets and multi-compounds in treating ICH. In these compounds, MOL000675 (degree =117) has the greatest number of targets, followed by MOL007022 (degree=114), MOL000131 (degree=111), MOL000098 (degree=107) and MOL004996 (Degree =106)), MOL001689 (degree =104), MOL003896 (XFZYD 5, degree =98), MOL004985 (XFZYD 2, degree =98), MOL002203 (XFZYD 23, degree =92). Most of these compounds are related to $\mathrm{ICH}$ apoptosis, inflammation and immune-related pathways. Parveen et al found that ACER2 was involved in sphingomyelin metabolism, which could catalyze ceramide hydrolysis to produce sphingosine, and sphingosine was further phosphorylated to produce sphingosine monophosphate [ 40 ]. ACER2 can control cells by controlling the relative levels of ceramide, sphingosine and sphingosine monophosphate, thus playing a role in the process of cell proliferation, aging and apoptosis. Studies show that lysophosphatidic acid (LPA) is a kind of lysophosphatide with wide biological activity, which can cause a variety of growth factor-like reactions that regulate cell proliferation, migration and survival. The role of other compounds in ICH treatment has been described in the chapters "Shared compounds of XFZYD Chinese herbal medicine" and "Specific compounds of XFZYD Chinese herbal medicine". These results proved the important role of these compounds in treating $\mathrm{ICH}$, and further confirmed the multi-compounds role of XFZYD in ICH treatment.

In the C-T network, the average target degree of different targets is 7.83. Most of the top 20 targets including MAPT, ALOX5AP, ESR2, ODC1 and ESR1, etc. which have the higher weights in the C-T network, are associated with immunity and inflammation and have been confirmed to be related to the pathogenesis of $\mathrm{ICH}$, and may indicate the potential mechanism of XFZYD on ICH. For example, 
Markoula et al found that ESR2 was significantly related to the risk of stroke [41]. ESR2 and COC exposure have synergistic effect in the occurrence of hemorrhagic stroke. Inflammation is the main pathogenic mechanism of atherosclerosis, and leukotriene is an important inflammatory mediator involved in the inflammatory reaction. Evans et al showed that 5-lipoxygenase (5-LO) activator protein (FLAP) was encoded and synthesized by ALOX5AP gene, which was necessary for 5-LO activation and leukotriene synthesis [ 42 ]. Jiang found that ODC1 could be used as a common target to control inflammatory reaction and could exert its inhibitory effect on macrophage inflammatory reaction and inhibit ROS-induced macrophage apoptosis [ 43 ]. These results suggest that XFZYD can treat ICH through the synergistic effect of regulating inflammation and immune function, which further confirms the multi-target effect of XFZYD in the treatment of ICH.

The main targets of the active compounds are often involved in HIF-1 signaling pathway(hsa04066), VEGF signaling pathway(hsa04370), MAPK signaling pathway (hsa04010), Alzheimer disease(hsa05010) and PI3K-Akt signaling pathway (HSA 04151). VEGF signaling pathway is very important for cell proliferation and growth. According to literatures, VEGF stimulates the expression level of the PI3K-Akt and MAPK-ERK proteins in the cerebral cortex of rats through phosphate Akt and activate MAPK-regulated ERK pathways, thus reducing the lesion volume of cerebral infarction and promoting angiogenesis in rats with cerebral infarction [ 44 ]. Studies have shown that PI3K is an important phosphatidylinositol kinase, which involve in cell growth and bone remodeling, and is an important antiapoptosis regulator. Activation of AKT can induce the phosphorylation of lipid substrate and can promote downstream protein kinase $B$, which can contribute to cell proliferation and differentiation [ 45]. The results show that the optimization strategy of $\mathrm{CHM}$ prescription combining effective space with $\mathrm{Cl}$ model is reliable, and the predicted CCG may play a therapeutic role by mediating various related ways.

\subsection{Selection and Validation of Effective Proteins}

Using weighted pathogenic gene-gene interactions and C-T network to construct compounds-targetspathogenic genes (C-T-P) network. This network is consisting 2,932 nodes and 48,980 edges. Degree of each node can be used to evaluate the importance of nodes in the network. The node with the degree which is greater than the average degree of all nodes in the network is considered to play a key role in the network structure and can be regarded as a central node. According to this rule, the transmitted nodes and their edges are retained in the C-T-P network, and defined as the effective space. 445 effective proteins were identified from the effective space. There are three kinds of effective proteins in the effective space. The first category of making a table is the direct interaction between pathogenic genes and compounds targets. We define this category as the basic effective compounds. The second category we defined as the targets specific interactions, which contain the interactions, only existed between the targets of compounds. The third category we defined as the pathogenic genes specific interactions, which mean the interactions linked the different pathogenic genes.

In order to further prove the reliability and accuracy of our model, we compare our proposed node importance calculation method with other commonly used node importance calculation methods,including degree, betweenness centrality, and clustering coefficient. We use our model and these 
models to obtain respective coefficient proteins and use these effective proteins to perform pathway and GO enrichment analysis,respectively, and then check the percentages of effective protein-enriched pathways and GO terms in intervention pathways and intervention GO terms, respectively. The higher the percentage, the higher the reliability and accuracy of the model. Results show that the percentage of effffective proteinenriched pathways found in our model in intervention pathway and intervention GO term is signifificantly higher than that in the degree model, betweenness centrality model, and clustering coeffiffifficient model. These results show that compared with other node importance models, our model has higher accuracy and better functional coverage (Figures 2).

We evaluated whether the targets of effective proteins screened from the effective space can cover the pathogenic genes of $\mathrm{ICH}$ at the functional level by enriched pathways. As result, targets of effective proteins have 172 enriched pathways $(p<0.05)$, while pathogenic genes can be enriched to 195 pathways $(p<0.05)$. It was found that effective protein enriched pathways covered $88.21 \%$ of pathogenic gene enriched pathways (Figures 3). In addition, in order to evaluate whether the effective space can be replaced by basic effective interactions, pathogenic genes specific interactions or compounds targets specific interactions, so as to further optimize. We analyzed the paths of basic effective interactions; pathogenic genes specific interactions and compounds targets specific interactions. The results showed that the coverage rates of the three enriched pathways were $87.18 \%, 75.38 \%$ and $20.00 \%$, respectively. These results validated the reliability and accuracy of our effective space selection method, and further proved that the effective protein selected in the effective space played leading roles in the pathogenesis of XFZYD. According to some literatures, PI3K/Akt/mTOR signaling pathway play important roles in cell proliferation and growth. The increase of Akt activity can reduce the expression of substrate p27kip1 in $\mathrm{ICH}[46$ ]. Therefore, ICH lymphocytes accumulate towards apoptosis or proliferation in $\mathrm{S}$ phase and G2/M phase. Previous studies proved that the abnormal activation of PI3K/AKT signaling pathway may be involved in the pathogenesis of $\mathrm{ICH}$ in $\mathrm{ICH}$ patients by up-regulating $\mathrm{CDKs}$ and down-regulating p27Kip1 and p21WAF1/CIP1 [47]. These results of KEGG enrichments above and reports showed that XFZYD is related to the pathogenesis of $\mathrm{ICH}$.

\subsection{Selection and Verification of CCG}

Establish Cl model, optimize effective compounds and get CCG, which lays a foundation for elucidating the underlying mechanism of XFZYD in treating ICH. According to the cumulative results of contributions, the top three compounds are EIC, quercetin and acacetin. The coverage rate of targets of wogonin and evofolinB to effective protein was $50.56 \%$. For further analysis, 43 compounds can contribute to the target coverage of $90.11 \%$ of effective proteins and are selected as CCG(Figures 4 ) The high targeted coverage rate of effective protein indicates that CCG may play a leading role in ICH treatment and produce joint effect.

We analyzed the function of XFZYD in the treatment of ICH base on the KEGG enriched pathways of CCG targets and ICH pathogenic genes. As result, we obtained 173 CCG targeted enrichment pathways $(p<0.05)$ and 195 pathogenic gene enrichment pathways $(p<0.05)$. It was found that CCG targeted 
enrichment pathway covered $88.72 \%$ of pathogenic gene enrichment pathways (Figures 5). The targets of CCG are mostly enriched in HIF-1 signaling pathway (hsa04066), VEGF signaling pathway (hsa04370), MAPK signaling pathway (hsa04010), Alzheimer disease (hsa05010) and PI3K-Akt signaling pathway (HSA 04151). VEGF signaling pathway (hsa04370) is very important for cell proliferation and growth. The results show that the optimization strategy of $\mathrm{CHM}$ prescription combining optimization space with $\mathrm{Cl}$ model is reliable, and the predicted CCG may play a therapeutic role by mediating various related ways. In order to verify whether our predicted CCG can represent all CT networks of XFZYD, two strategies are used to verify the reliability and accuracy of CCG. The first is based on the coverage of CCG pathogenic genes, which is defined as the percentage of the number of pathogenic genes in the network and C-T network; the second is based on network topology. Evaluate whether the number of CCG pathogenic genes is nearly equal to the number of $\mathrm{ICH}$ pathogenic genes in these 2 strategies, we found that C-T network contains 333 pathogenic genes. There are 298 pathogenic genes in CCG gene sequence. Compared with C-T network, the number of pathogenic genes in this network reached $89.49 \%$, which confirmed that the predicted CCG had higher disease coverage rate. These results indicate that CCG has a high degree of agreement with $\mathrm{C}-\mathrm{T}$ network in the number and coverage of pathogenic genes.

\subsection{Function Analysis of CCG Targets}

GO enrichment analysis was performed using the R software clusterProfiler package to identify the biological functions of the main targets with $p$ values $<0.05$. To further profile the combined effects of XFZYT, all targets that interacted with CFCG in XFZYT were enriched by GO enrichment analysis (Figures 6 ).

GO analysis showed that the regulatory targets of XFZYT were abundantly expressed in biological processes related to inflammatory response, oxidative stress and apoptosis. For example, leukocyte activation involved in inflammatory responses (G0:0002758), the production of molecular mediators involved in inflammatory responses (G0:0070498), and inflammatory responses to antigen stimulation (G0:0031349). These results confirmed that XFZYT could treat ICH by reducing inflammatory reaction, reducing oxidative stress and inhibiting apoptosis.

\subsection{Pathway Enrichment Analysis of CCG}

ICH-related pathways can be decomposed into functional modules such as apoptosis, inflammation, and oxidative stress. Increasing evidences confirmed that the PI3K-Akt signaling pathway (hsa04151), JAKSTAT Signaling pathway (HSA04630), NF-kappa B signaling pathway (hsa04064), and IL-17 signaling pathway (hsa04657) respond to these functional modules. For example, PI3K-Akt signaling pathway (hsa 04151) has been reported to be involved in the inhibition of apoptosis, cell proliferation, and expression of inflammatory cytokines. JAK-STAT signaling pathway (HSA04630) is involved in pathophysiological changes in damaged peripheral brain tissue after cerebral hemorrhage, leading to brain tissue edema. NF-kappa B signaling pathway (hsa04064) is a general name of a family of transcription factors that function as dimers to regulate genes of immunity, inflammation and cell survival. IL-17 signaling pathway (hsa04657), a subset of cytokines composed of IL-17A-F, plays an 
important role in acute and chronic inflammatory responses. For exploring the mechanism of XFZYT in the treatment of $\mathrm{ICH}$ at the system level, we constructed a comprehensive signaling pathway using two important molecular pathways (Figures 7 ), the PI3K-Akt signaling pathway and the MAPK signaling pathway. This pathway plays an important role in the treatment of cerebral hemorrhage. We treat the first three columns as upstream locations of the integrated pathway, and the remaining columns as downstream locations of the integrated pathway. Among them, PI3K-Akt signaling pathway (hsa04151) is one of the main pathways for XFZYT to treat ICH. XFZYT regulated 12 targets upstream of the PI3K-Akt signaling pathway (hsa04151), such as KDR, VEGFA, and PTK2and 35 targets downstream, such as Akt3 and CHUK. Downstream targets account for more than 70\%. XFZYT may activate the downstream PI3K and AKT proteins through the upstream KDR, resulting in the cascade amplification of downstream PIK3CA, Akt3, and GSK3B, which is closely related to ICH cell proliferation and protein synthesis. Most of the targets of XFZYT in the regulation of PI3K-Akt signaling pathway are located in the downstream of this pathway, such as Akt3 and PIK3CA. After the PI3K/AKT signaling pathway is activated, it can regulate the apoptosis of other downstream target proteins to exert biological effects, playing a key role in the treatment of $\mathrm{ICH}$.

\subsection{Experimental Validation In Vitro}

The effects of acacetin, queretin, luteolin on OGD model were evaluated. Compared with control group, the cell viability of OGD groups were significantly reduced. Compared with control group, the effect of cell viability was significantly decreased by $33.48 \%$ in the hypoxia treated cells. However, Acacetin $(80,120$, 160 and $200 \mu \mathrm{M}$ ) markedly increased the cell viability level by $16.00 \%, 18.10 \%, 20.73 \%$ and $12.42 \%$

(Figures $8 \mathrm{~A}$ ). Compared with control group, the effect of cell viability was significantly decreased by $44.38 \%$ in the hypoxia treated cells. However, Queretin $(120,160$ and $200 \mu \mathrm{M})$ markedly increased the cell viability level by $28.77 \%, 29.40 \%$ and $32.66 \%$ (Figures $8 B$ ). Compared with control group, the effect of cell viability was significantly decreased by $32.59 \%$ in the hypoxia treated cells. However, Luteolin $(40,80$, 120,160 and $200 \mu \mathrm{M}$ ) markedly increased the cell viability level by $15.82 \%, 22.52 \%, 19.42 \%, 24.84 \%$ and $28.35 \%$ (Figures $8 \mathrm{C}$ ). The above results demonstrated that acacetin, queretin and luteolin possessed protect effect in hypoxia treated HT22 cells.

\section{Discussions}

In the process of treating complex diseases, Chinese medicine prescriptions usually exert their functional effects according to compatibility principles. Chinese medicinal prescriptions composed of different herbal medicines often contain hundreds of chemical compounds. Some of these chemicals have a synergistic therapeutic effect, while some may have an antagonistic therapeutic effect. How to figure out the synergistic or antagonistic effects of these chemical compounds and optimize the core therapeutic chemical compounds is the main purpose of prescription optimization.

In addition, in the process of treating diseases, the action form of prescriptions in TCM is relatively complex, and usually acts in the form of multi-compounds multi-targets and multi-pathways, which 
constitutes a multi-dimensional regulatory network. How to find the core functional compound groups in these regulatory networks and analyze the mechanism is one of the key ways for us to understand the mechanism of prescriptions in the treatment of complex diseases.

Node importance is one of the most important ways to depict the network. In this research, we designed a new node importance calculation method and validated the accuracy and reliability. Based on this new node importance calculation method, optimization space was constructed. Potential effective proteins were screened out based on the optimization space, and then the key constituent groups corresponding to the effective proteins were obtained using the reverse programming algorithm. We analyzed the enrichment pathways of these CCG and found that the target enrichment pathways of these CCG accounted for $90.11 \%$ of the enrichment pathways of pathogenic genes. These results fully proved the accuracy and reliability of our computational model for screening key compound groups based on the optimization space. Our proposed screening strategy consists of two levels of design. The first level of design is to construct the optimization space based on the node importance calculation method, and verify that the information of disease-related genes will be preserved as much as possible in this step as the core protein or functional protein in the optimization space. In the second step, potential CCG are reversely screened based on the core proteins or functional proteins, and further pathway enrichment analysis is performed on the functional compound group for verification. The results of this dual model and verification fully demonstrate the reliability of our integrated analysis model, and provide methodological reference for other prescription optimization in TCM.

\section{Abbreviations}

Contribution index: Cl; Drug-likeness: DL; Gene Ontology: GO; Key group of effective compounds: CCG; Kyoto Encyclopedia of Genes and Genomes: KEGG; Xue Fu Zhu Yu Decoction: XFZYD; Oral bioavailability: OB; Protein-protein interactions: PPI; Hypertensive Cerebral Hemorrhage: ICH; Traditional Chinese medicine: $\mathrm{CHM}$.

\section{Declarations}

\section{- Ethics approval and consent to participate}

Not applicable.

\section{- Consent to publish}

Agree to publish the paper without dispute.

\section{- Availability of data and materials}

The datasets used or analysed during the current study are available from the corresponding author.

Similarity Ensemble Approach 囚https://sea.bkslab.org/ 
GeneCards database \https://www.genecards.org/

STRING囚https://cn.string-db.org/

BioGRID 『https://thebiogrid .org/

HPRD囚http://www.hprd.org/

\section{- Competing interests}

The authors declare no conflict of interest.

\section{- Funding}

This study is financially supported by the Startup fund from Scientific Research Project of Guangdong Provincial Administration of Traditional Chinese Medicine (No. 20181111), the Startup fund from Guangzhou Mulicipal Science and Technology Bureau (No. 202002030221), the Natural Science Foundation Council of China [grant No. 31501080].

\section{- Authors' Contributions}

XDJ, ZZ and WKX provided the concept and designed the study, XDJ, ZZ, PZZ and SYB conducted the analyses and wrote the manuscript. GDG, XDJ and GY participated in data analysis. ZZ and ZJL carried out experimental validation. GDG and ZZ contributed to revising and proof-reading the manuscript. All authors read and approved the final manuscript.

\section{- Acknowledgements}

Not applicable.

\section{References}

1. Lian,L.F., \& Zhu,S.Q. (2019).Interpretation in the update of Chinese guidelines for diagnosis and treatment of acute intracerebral hemorrhage 2019. Chinese Journal of Neurology(12),985-986-987988. doi:10.3760/cma.j.issn.1006-7876.2019.12.001.

2. Wang,Y.J., Li,Z.X., Gu,H.Q., Zhai,Q., ... \&Zhao,J.Z. (2020).China Stroke Report 2019 (Chinese version). Chinese Journal of Stroke(12),1251-1263. doi:CNKI:SUN:ZUZH.0.2020-12-001.

3. Cui, H., Liu, T., Li, P., Yang, A., Zhou, H., Luo, J., Hu, E., Hu, W., Wang, Y., \& Tang, T. (2018). An Intersectional Study of LncRNAs and mRNAs Reveals the Potential Therapeutic Targets of Buyang Huanwu Decoction in Experimental Intracerebral Hemorrhage. Cellular physiology and biochemistry: international journal of experimental cellular physiology, biochemistry, and pharmacology, 46(5), 2173-2186. https://doi.org/10.1159/000489547 
4. Huang, Q., Lan, T., Lu, J., Zhang, H., Zhang, D., Lou, T., Xu, P., Ren, J., Zhao, D., Sun, L., Li, X., \& Wang, J. (2018). DiDang Tang Inhibits Endoplasmic Reticulum Stress-Mediated Apoptosis Induced by Oxygen Glucose Deprivation and Intracerebral Hemorrhage Through Blockade of the GRP78-IRE1/PERK Pathways. Frontiers in pharmacology, 9, 1423. https://doi.org/10.3389/fphar.2018.01423

5. Shen,Y., \& Luo,L.L. (2015). Clinical observation on patients with hypertensive cerebral hemorrhage treated with modified Xuefu Zhuyu Decoction combined with western medicine. Journal of Emergency in Traditional Chinese Medicine(02),337-339. doi:.10.3969/j.issn.1004745X.2015.02.059

6. Li,Y.C., \& Li,Y.Q. (2009).Effects of Xuefu Zhuyu Decoction on Serum Asymmetric Dimethylarginine in Atherosclerosis Rats. GUIDE OF CHINA MEDICINE(03),5-6. doi:CNKI:SUN:YYXK.0.2009-03-005.

7. Lin,J.M., Zhou,H.T., Zheng,L.P., Zhou,J.H., Zhao,J.Y., Zhuang,Q.C ... \& Wang ,R.G. (2009).Effect of Xuefu Zhuyu Decoction on Secretion of VEGF and PDGF in Human Umbilical Vein Endothelial Cells. CHINESE JOURNAL OF INTEGRATIVE MEDICINE ON CARDIO-/CEREBROVASCULAR DISEASE(10),1182-1184. doi:CNKI:SUN:ZYYY.0.2009-10-031.

8. Wang, C., Ren, Q., Chen, X. T., Song, Z. Q., Ning, Z. C., Gan, J. H., Ma, X. L., Liang, D. R., Guan, D. G., Liu, Z. L., \& Lu, A. P. (2018). System Pharmacology-Based Strategy to Decode the Synergistic Mechanism of Zhi-zhu Wan for Functional Dyspepsia. Frontiers in pharmacology, 9, 841. https://doi.org/10.3389/fphar.2018.00841

9. Wang, K. X., Gao, Y., Lu, C., Li, Y., Zhou, B. Y., Qin, X. M., Du, G. H., Gao, L., Guan, D. G., \& Lu, A. P. (2020). Uncovering the Complexity Mechanism of Different Formulas Treatment for Rheumatoid Arthritis Based on a Novel Network Pharmacology Model. Frontiers in pharmacology, 11, 1035. https://doi.org/10.3389/fphar.2020.01035

10. Gao, Y., Wang, K. X., Wang, P., Li, X., Chen, J. J., Zhou, B. Y., Tian, J. S., Guan, D. G., Qin, X. M., \& Lu, A. P. (2020). A Novel Network Pharmacology Strategy to Decode Mechanism of Lang Chuang Wan in Treating Systemic Lupus Erythematosus. Frontiers in pharmacology, 11, 512877. https://doi.org/10.3389/fphar.2020.512877

11. Liu, T., Xu, P., Qi, S., Ke, S., Hu, Q., Zhao, P., \& Li, J. (2021). Network Pharmacology-Based Mechanistic Investigation of Jinshui Huanxian Formula Acting on Idiopathic Pulmonary Fibrosis. Evidence-based complementary and alternative medicine : eCAM, 2021, 8634705.

https://doi.org/10.1155/2021/8634705

12. He, D., Huang, J. H., Zhang, Z. Y., Du, Q., Peng, W. J., Yu, R., Zhang, S. F., Zhang, S. H., \& Qin, Y. H. (2019). A Network Pharmacology-Based Strategy For Predicting Active Ingredients And Potential Targets Of LiuWei DiHuang Pill In Treating Type 2 Diabetes Mellitus. Drug design, development and therapy, 13, 3989-4005. https://doi.org/10.2147/DDDT.S216644

13. Szklarczyk D, Gable AL, Lyon D, et al. STRING v11: protein-protein association networks with increased coverage, supporting functional discovery in genome-wide experimental datasets. Nucleic Acids Res. 2019;47(D1):D607-D613. doi:10.1093/nar/gky1131 
14. Ru, J., Li, P., Wang, J., Zhou, W., Li, B., Huang, C., Li, P., Guo, Z., Tao, W., Yang, Y., Xu, X., Li, Y., Wang, Y., \& Yang, L. (2014). TCMSP: a database of systems pharmacology for drug discovery from herbal medicines. Journal of cheminformatics, 6, 13. https://doi.org/10.1186/1758-2946-6-13

15. Xue, R., Fang, Z., Zhang, M., Yi, Z., Wen, C., \& Shi, T. (2013). TCMID: Traditional Chinese Medicine integrative database for herb molecular mechanism analysis. Nucleic acids research, 41 (Database issue), D1089-D1095. https://doi.org/10.1093/nar/gks1100

16. Chen C. Y. (2011). TCM Database@Taiwan: the world's largest traditional Chinese medicine database for drug screening in silico. PloS one, 6(1), e15939. https://doi.org/10.1371/journal.pone.0015939

17. Xu, X., Zhang, W., Huang, C., Li, Y., Yu, H., Wang, Y., Duan, J., \& Ling, Y. (2012). A novel chemometric method for the prediction of human oral bioavailability. International journal of molecular sciences, 13(6), 6964-6982. https://doi.org/10.3390/ijms13066964

18. Wang, Z., Liang, L., Yin, Z., \& Lin, J. (2016). Improving chemical similarity ensemble approach in target prediction. Journal of cheminformatics, 8, 20. https://doi.org/10.1186/s13321-016-0130-x

19. Liu, X., Vogt, I., Haque, T., \& Campillos, M. (2013). HitPick: a web server for hit identification and target prediction of chemical screenings. Bioinformatics (Oxford, England), 29(15), 1910-1912. https://doi.org/10.1093/bioinformatics/btt303

20. Gfeller, D., Grosdidier, A., Wirth, M., Daina, A., Michielin, O., \& Zoete, V. (2014). SwissTargetPrediction: a web server for target prediction of bioactive small molecules. Nucleic acids research, 42(Web Server issue), W32-W38. https://doi.org/10.1093/nar/gku293

21. Yu, G., Wang, L. G., Han, Y., \& He, Q. Y. (2012). clusterProfiler: an R package for comparing biological themes among gene clusters. Omics : a journal of integrative biology, 16(5), 284-287. https://doi.org/10.1089/omi.2011.0118

22. Draghici, S., Khatri, P., Tarca, A. L., Amin, K., Done, A., Voichita, C., Georgescu, C., \& Romero, R. (2007). A systems biology approach for pathway level analysis. Genome research, 17(10), 1537-1545. https://doi.org/10.1101/gr.6202607

23. Morán, J., Perez-Basterrechea, M., Garrido, P., Díaz, E., Alonso, A., Otero, J., Colado, E., \& González, C. (2017). Effects of Estrogen and Phytoestrogen Treatment on an In Vitro Model of Recurrent Stroke on HT22 Neuronal Cell Line.Cellular and molecular neurobiology, 37(3), 405-416.

24. Zhu,B., Chen,J., Qin,H.F., Li,H... \& Chen,D.F. (2012).Early Changes of Pathology and Cognitive Behavior in APP/PS1 Transgenic Mice with Alzheimer's Disease. Journal of Guangzhou University of Traditional Chinese Medicine(02),193-196+226. doi:10.13359/j.cnki.gzxbtcm.2012.02.027.

25. Fu,J.Y., Tang,L., \& Fan,D.S. (2019).Genetic variants in NOTCH3 associated with spontaneous intracerebral hemorrhage. Chinese Journal of the Frontiers of Medical Science(Electronic Version) (10),11-18. doi:CNKI:SUN:YXQY.0.2019-10-004.

26. Wang,L.S., Zhang,Y.Q., Feng,Z., Ying,X.W., Wang,X.J., \& Huo,X.L. (2017).Effects of oxidative stress on neuronal apoptosis and protein expression of c-myc after intracerebral hemor rhage. Chinese Journal of General Practice(04),608-610+676. doi:10.16766/j.cnki.issn.1674-4152.2017.04.020. 
27. Qian,H., Liu,L.J., Li,Y.C., Xie,M., Wu,H., Liu,S.X., \& Wu,B. (2016).EGFR siRNA inhibits activation of astrocytes derived from rats through blockade of STAT3 phosphorylation. Chinese Pharmacological Bulletin(02),216-222. doi:10.3969/j.issn.1001-1978.2016.02.014

28. Chen,Y.H., Zhao,X.X., Wang,W.Q., \& Duan,T.X. (2009).Simultaneous Determination of Glycyrrhizin,Liquiritin and Isoliquritigenin in Licorice by HPLC. CHINESE JOURNAL OF INFORMATION ON TRADITIONAL CHINESE MEDICINE(08),52-54. doi:10.3969/j.issn.1005-5304.2009.08.024.

29. Xie,J.J., Yu,Y., Wang.Y.T., \& Li,S.P. (2007).Simultaneous HPLC determination of 6 components in Angelica sinensis. CHINESE JOURNAL OF PHARMACEUTICAL ANALYSIS(09),1314-1317. doi:CNKI:SUN:YWFX.0.2007-09-001.

30. Li,W.M., Zhao,Y.R., Yang,Y.Y., Zhang,Z.Q., Lai,J.Y., \& Zhang,L. (2011).RP- HPLC with UV switch determination of 9 components in white peony root pieces. Chinese Journal of Pharmaceutical Analysis(12),2208-2212. doi:10.16155/j.0254-1793.2011.12.010.

31. Zhan,Z.B., Deng,Z.B., Xiong,Y., Ding,B.Q., \& Deng,M.Z. (2015).Simultaneous determination of six ingredients in Aurantii Fructus Immaturus by HPLC. Chinese Journal of Hospital Pharmacy(12),10801082. doi:10.13286/j.cnki.chinhosppharmacyj.2015.12.06.

32. Li,Z.G., Guo,X.H., Cao,L.P., \& Wang,X.Y. (2013). Determination of Nucleosides Compounds in Walnut Kernel from 10 Different Regions. Chinese Journal of Experimental Traditional Medical Formulae (10),145-148. doi:.10.11653/syfj2013100145

33. Wu, Y., Huai,J., \& Zhou, P. (2019). Determination of five components in Safflower. Asia-Pacific Traditional Medicine (11),82-87. doi:.10.11954/ytctyy.201911024

34. Liu,J.L, Fan,Q.J., Zheng,S.L., Tan,J., Zhou,J., Yuan,J.C., ... \& Kong,F.L. (2014).Quantitative determination of 5 active ingredients in different harvest periods of Ligusticum chuanxiong by HPLC. Chinese Journal of Traditional Chinese Medicine (09),1650-1655. doi:.10.4268/cjcmm20140921

35. Wu,T. (2016). Simultaneous HPLC Determination of Content of Catalpol and Verbascoside in Rehmannia. Heilongjiang Science and Technology Information(19),106. doi:10.3969/j.issn.16731328.2016.19.103.

36. Wang,F., Lu,D., Liu,C., Chen,X.H., Bi,K.S., \& Li,Q. (2011).RP-HPLC determination of 3 compounds of triterpenoid saponins in Platycodon grandiflorum. CENTRAL SOUTH PHARMACY(05),329-331. doi:10.3969/j.issn.1672-2981.2011.05.003.

37. Liang,X.K., Lei,J.W., Gong,H.Y., Tang,W.W., Ji,L., Xie,C.X., \& Liu,D.F. (2019). Simultaneous Determination of the Contents of Five Components in Achyranthis Bidentatae Radix and Rhizome by HPLC. Herbs (07), 1578-1583. DOI: 10.13863/J. ISSN 1001-4454.2019.07.024.

38. Hu,Y.J., Pu,J.B., Liang,W.Q., Zheng,J.X., \& Wei,K.M. (2011).Determination of Saikosaponin d and Total Saikosaponin Contents in Radix Bupleur Extract.. CHINESE JOURNAL OF TRADITIONAL MEDICAL SCIENCE AND TECHNOLOGY(01),46-47+56. doi:CNKI:SUN:TJYY.0.2011-01-035.

39. Xu,L.J.,Y.B., \& Li,B.L. (2017).Study on the effect of quercetin on inflammation and apoptosis in experimental intracerebral hemorrhage model in rats. Chinese Journal of Neurotraumatic Surgery (Electronic Edition)(02),93-97. doi:CNKI:SUN:SJCW.0.2017-02-010. 
40. Parveen, F., Bender, D., Law, S. H., Mishra, V. K., Chen, C. C., \& Ke, L. Y. (2019). Role of Ceramidases in Sphingolipid Metabolism and Human Diseases. Cells, 8(12), 1573.

https://doi.org/10.3390/cells8121573

41. Markoula, S., Milionis, H., Lazaros, L., Spengos, K., Vassilopoulou, S., Chatzistefanidis, D., Kargiotis, O., Georgiou, I., \& Kyritsis, A. P. (2012). Associations of ESR2 Alul (G/A) polymorphism with ischemic stroke in Caucasians. Journal of the neurological sciences, 316(1-2), 126-130.

https://doi.org/10.1016/j.jns.2012.01.007

42. Evans, J. F., Ferguson, A. D., Mosley, R. T., \& Hutchinson, J. H. (2008). What's all the FLAP about?: 5lipoxygenase-activating protein inhibitors for inflammatory diseases. Trends in pharmacological sciences, 29(2), 72-78. https://doi.org/10.1016/j.tips.2007.11.006

43. Jiang, F., Gao, Y., Dong, C., \& Xiong, S. (2018). ODC1 inhibits the inflammatory response and ROSinduced apoptosis in macrophages. Biochemical and biophysical research communications, 504(4), 734-741. https://doi.org/10.1016/j.bbrc.2018.09.023

44. Chen, J., Zhang, X., Liu, X., Zhang, C., Shang, W., Xue, J., Chen, R., Xing, Y., Song, D., \& Xu, R. (2019). Ginsenoside Rg1 promotes cerebral angiogenesis via the PI3K/Akt/mTOR signaling pathway in ischemic mice. European journal of pharmacology, 856, 172418.

https://doi.org/10.1016/j.ejphar.2019.172418

45. Chen, S., Peng, J., Sherchan, P., Ma, Y., Xiang, S., Yan, F., Zhao, H., Jiang, Y., Wang, N., Zhang, J. H., \& Zhang, H. (2020). TREM2 activation attenuates neuroinflammation and neuronal apoptosis via $\mathrm{PI3K} /$ Akt pathway after intracerebral

46. Shen, X., Xu, X., Xie, C., Liu, H., Yang, D., Zhang, J., Wu, Q., Feng, W., Wang, L., Du, L., Xuan, L., Meng, C., Zhang, H., Wang, W., Wang, Y., Xie, T., \& Huang, Z. (2020). YAP promotes the proliferation of neuroblastoma cells through decreasing the nuclear location of p27Kip1 mediated by Akt. Cell proliferation, 53(2), e12734. https://doi.org/10.1111/cpr.12734

47. Liu, H., Zeng, L., Yang, K., \& Zhang, G. (2016). A Network Pharmacology Approach to Explore the Pharmacological Mechanism of Xiaoyao Powder on Anovulatory Infertility. Evidence-based complementary and alternative medicine : eCAM, 2016, 2960372.

https://doi.org/10.1155/2016/2960372

\section{Figures}




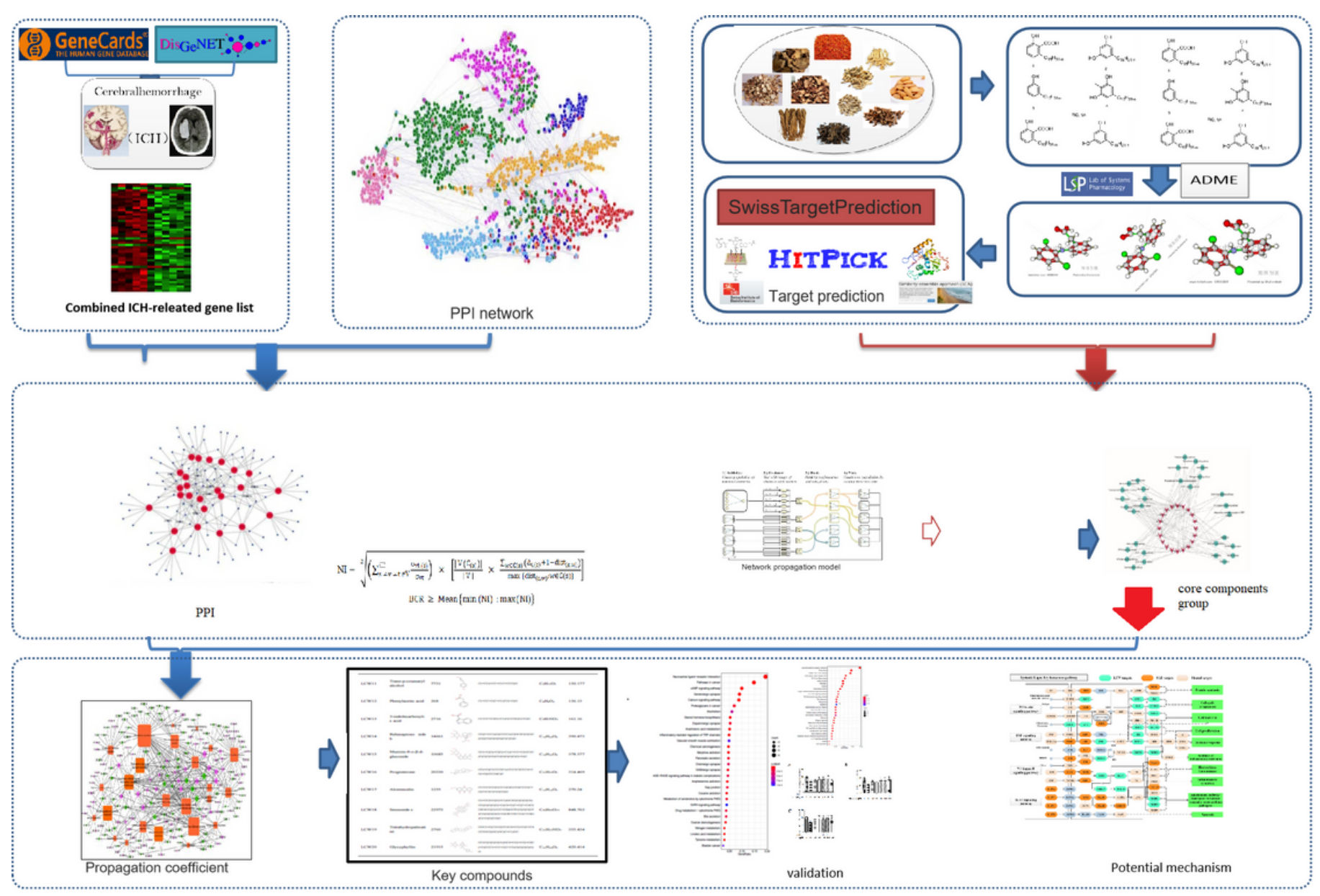

Figure 1

The work scheme of system pharmacology approach. 
A

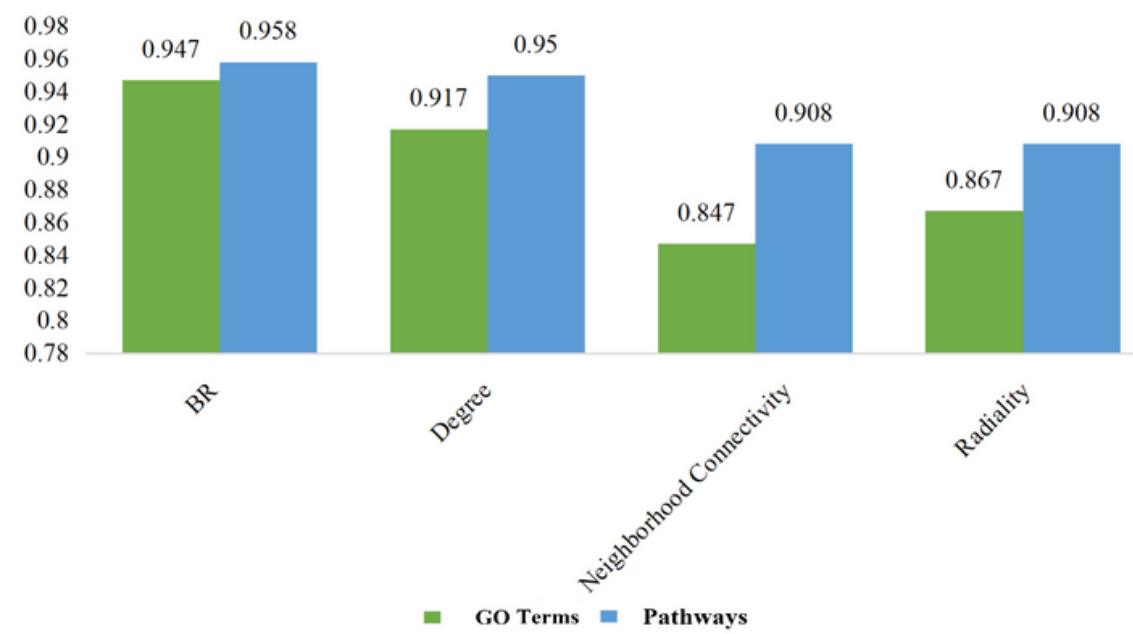

B

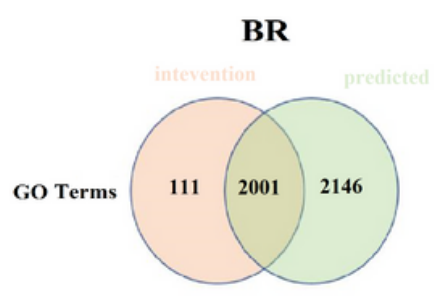

Degree
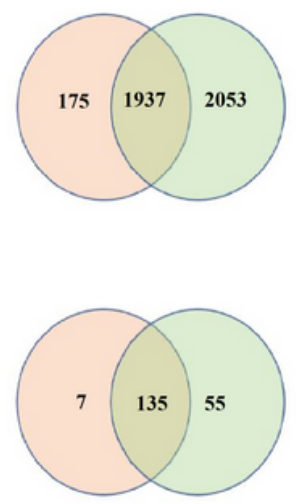

Neighborhood Connectivity

Radiality
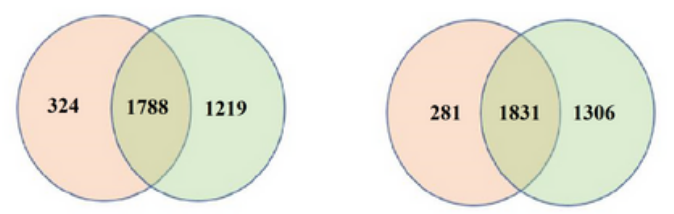

C
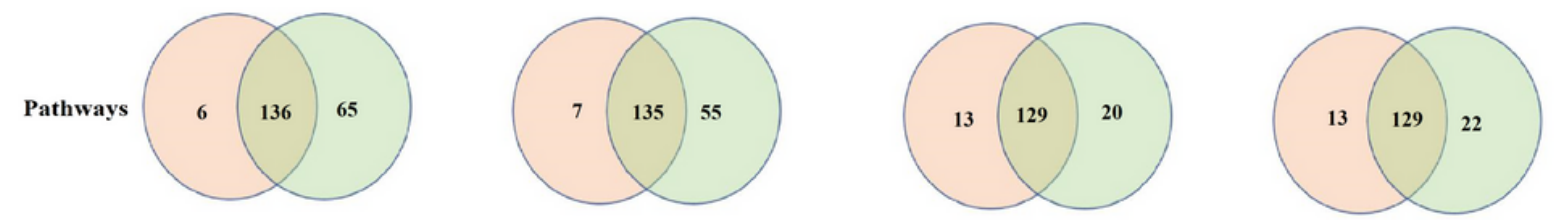

Figure 2

Compare our proposed model with other widely used models. (a) Venn diagrams display the number of overlapped GO terms of four models with main intervention GO terms. (b) Venn diagrams display the number of overlapped pathways of four models with intervention pathways, respectively. (c) Comparison of our model with other models on the intervention pathways and GO terms. 
A

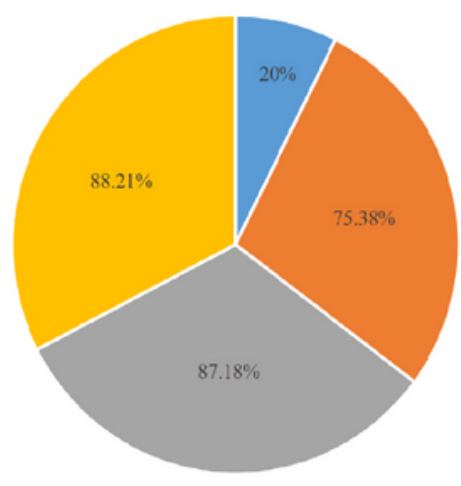

- drug target specific | disease specific $\mid$ common targets || effective proteins

B

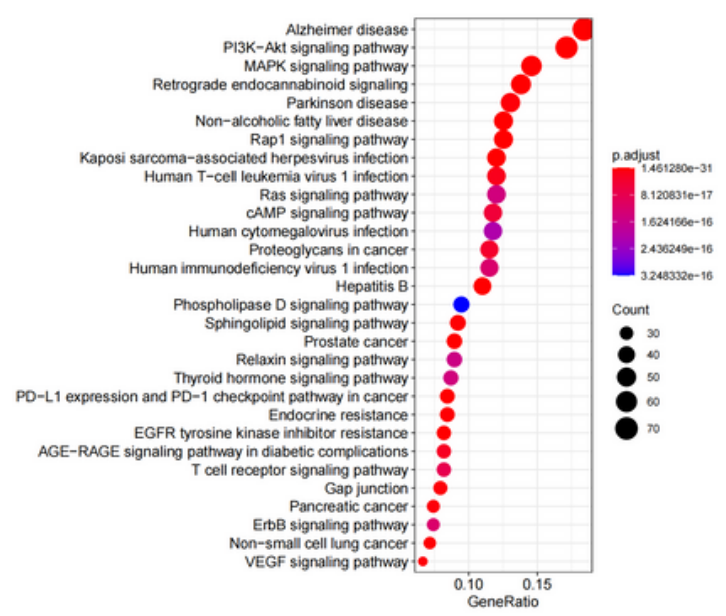

C

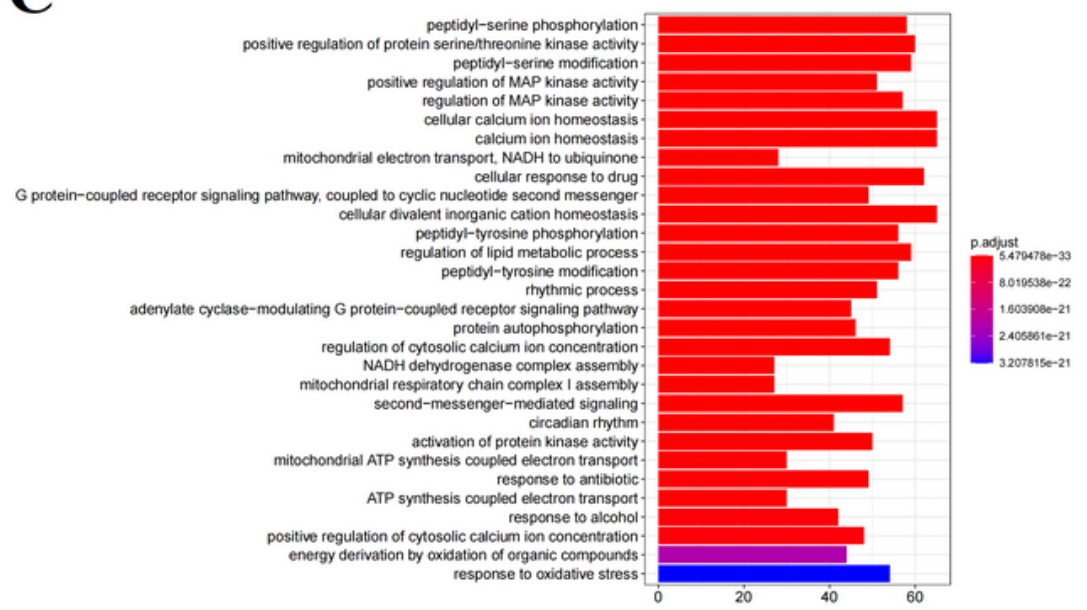

Figure 3

Effective space validation. The coverage rate of effective proteins, common targets, disease-specific targets, and drug-specific targets-enriched pathways compared with pathogenetic geneenriched pathways of ICH (A). KEGG (B) and GO (C) enrichment processing of effective protein. 


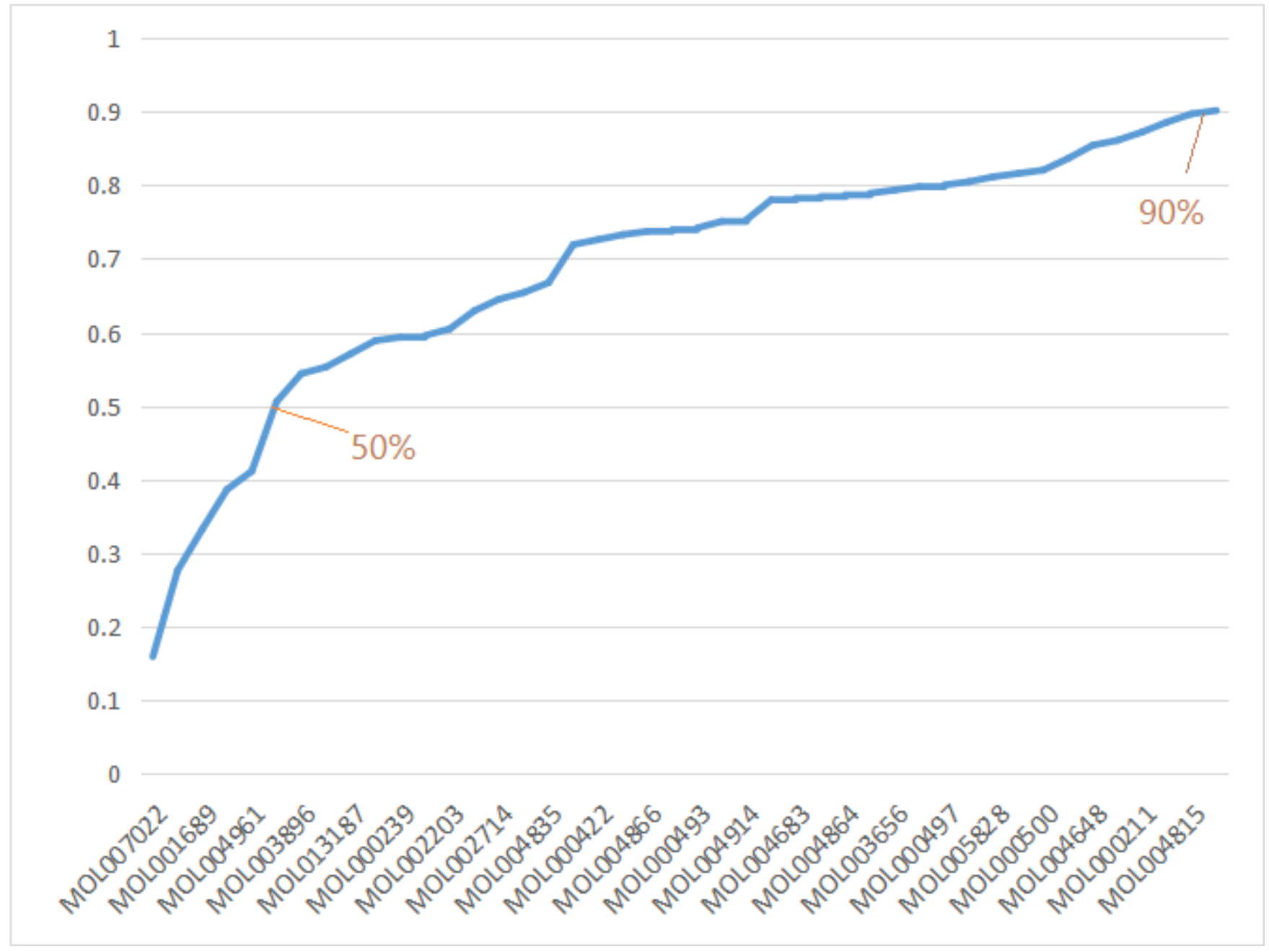

Figure 4

The accumulative CCG of effective compounds in effective space. 


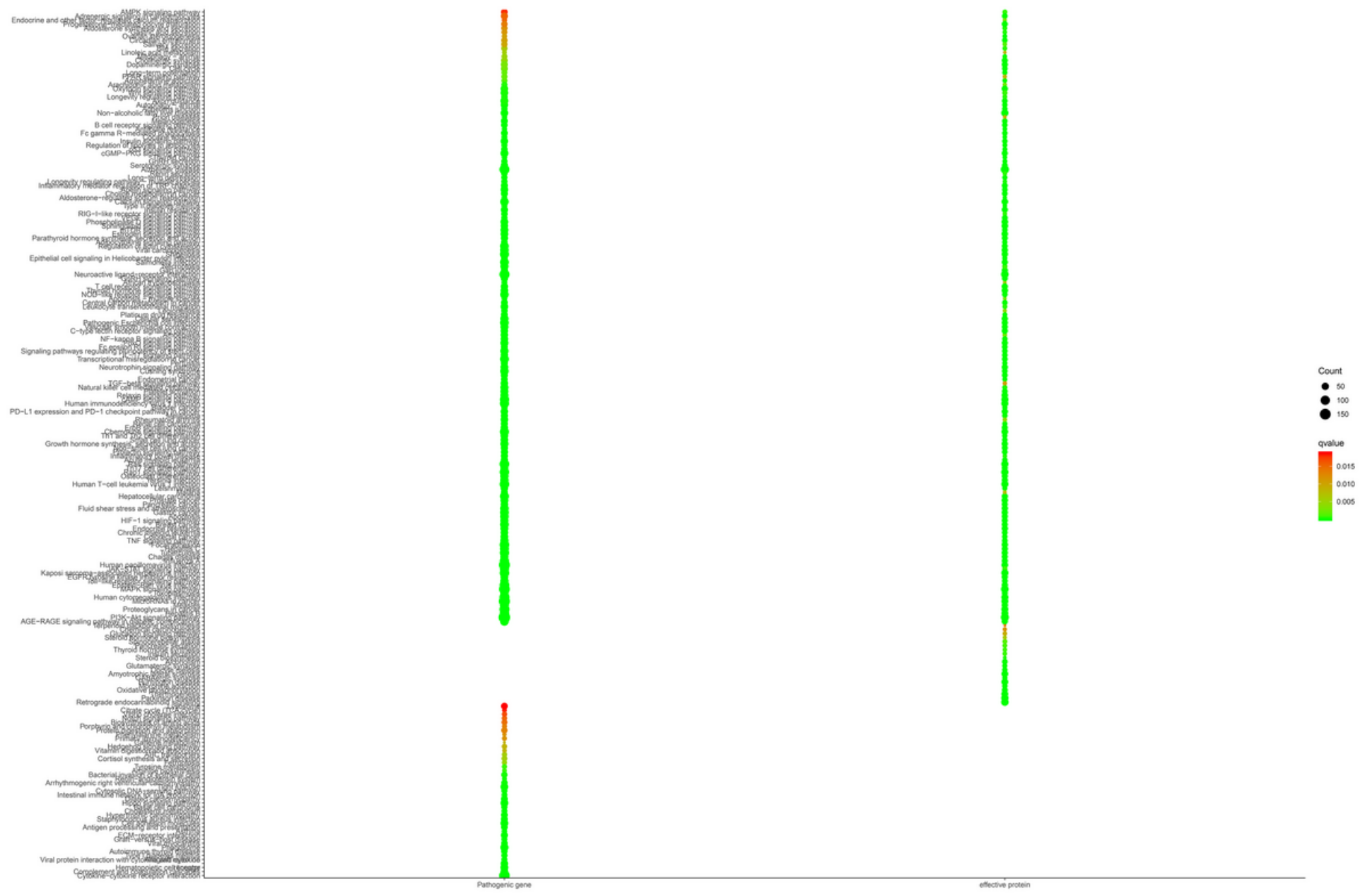

Figure 5

The functional similarity visualized between CCG targets and ICH pathogenic genes-enriched pathways. 
A

Barplot of GOMF Enrichment

oxidoreductase activity, acting on $\mathrm{NAD}(\mathrm{P}) \mathrm{H}$, quinone or similar compound as acceptor $\mathrm{NADH}$ dehydrogenase activity $\mathrm{NADH}$ dehydrogenase (ubiquinone) activity

$\mathrm{NADH}$ dehydrogenase (quinone) activity

oxidoreductase activity, acting on $\mathrm{NAD}(\mathrm{P}) \mathrm{H}$ protein tyrosine kinase activity nuclear receptor activity

ligand-activated transcription factor activity transmembrane receptor protein tyrosine kinase activity transmembrane receptor protein kinase activity protein serine/threonine kinase activity steroid hormone receptor activity oxidoreductase activity, acting on paired donors, with incorporation or reduction of molecular oxygen monocarboxylic acid binding oxidoreductase activity, acting on the $\mathrm{CH}-\mathrm{OH}$ group of donors, NAD or NADP as acceptor oxidoreductase activity, acting on $\mathrm{CH}-\mathrm{OH}$ group of donors monooxygenase activity bioactive lipid receptor activity cyclin-dependent protein kinase activity carbonate dehydratase activity

B

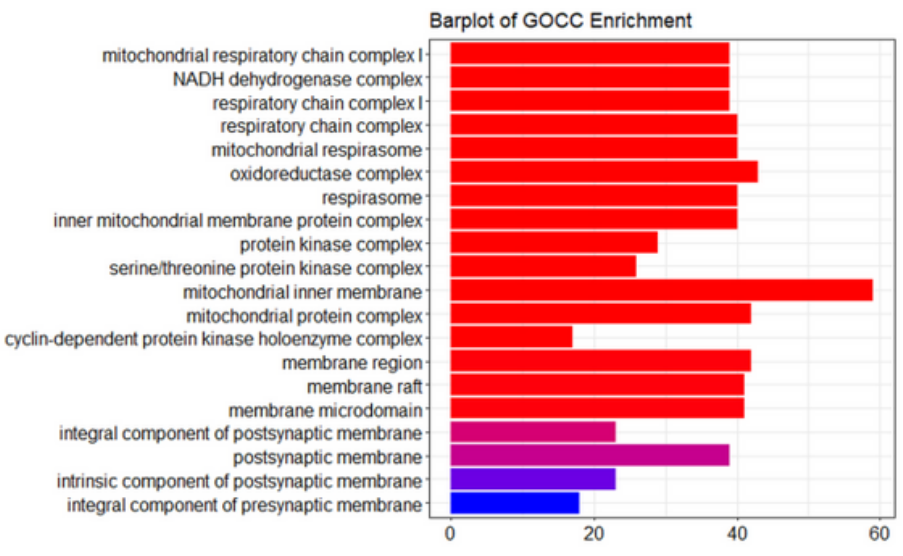

\section{C}

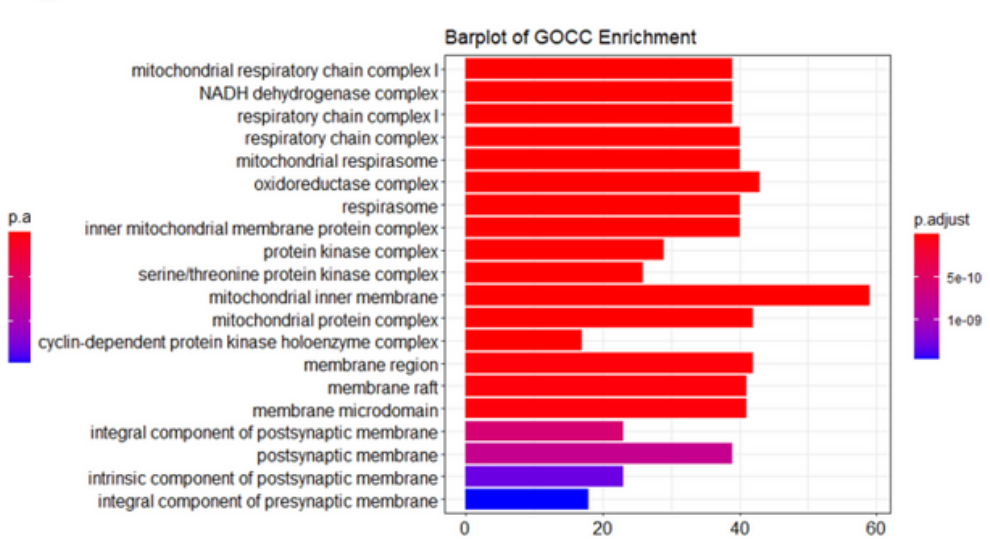

\section{Figure 6}

\section{GO enrichment processing of CCG targets.}




\section{XFZYT targets}

Shared targets between XFZYT and pathogenic genes of ICH

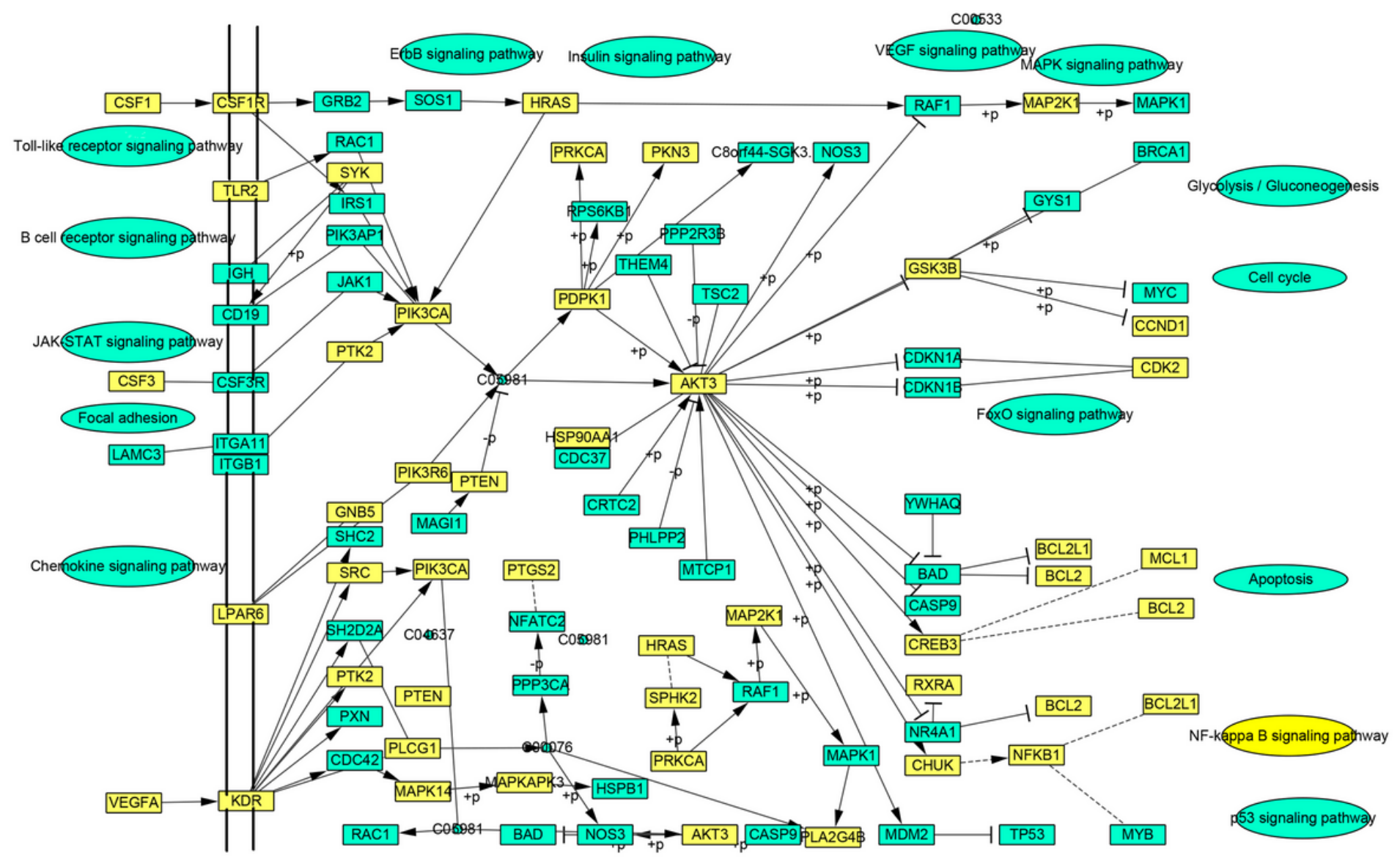

Figure 7

Distribution of targets of XFZYT on the compressed ICH pathway. 
A

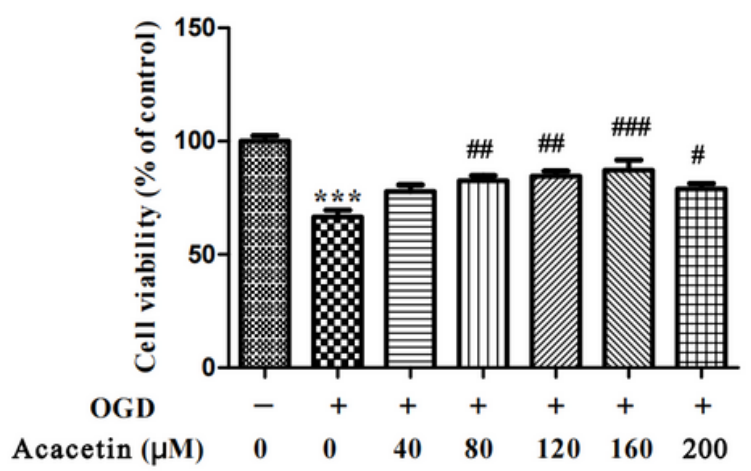

C

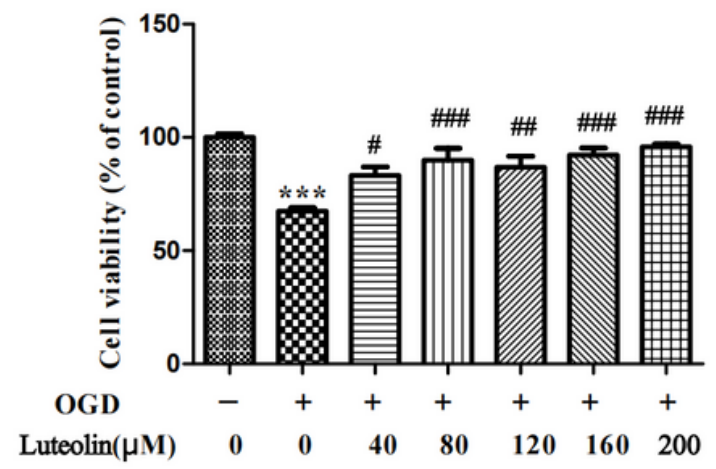

B

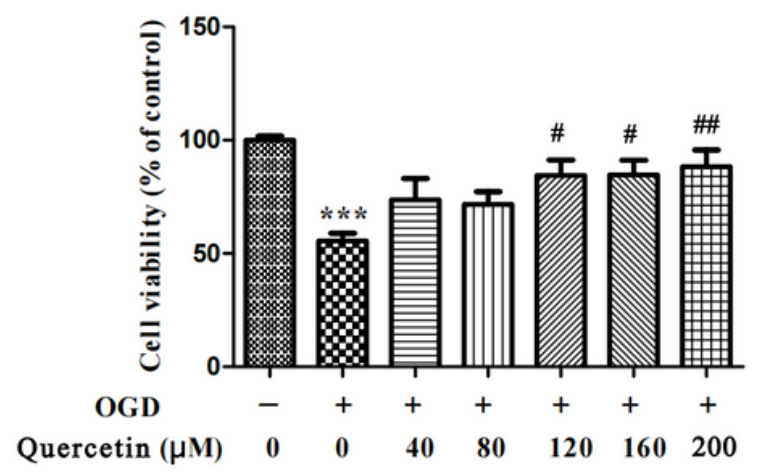

Figure 8

Effects of acacetin (A), queretin (B), and luteolin (C) on cell viabilities. *** $<0.001$ compared with control group. \#p < 0.05, \#p < 0.05, \#\#\#p < 0.001 compared with the OGD group. 